\title{
KULEUVEN
}

\section{Option prices and model-free measurement of implied herd behavior in stock markets}

Daniël Linders, Jan Dhaene and Wim Schoutens 


\title{
Option prices and model-free measurement of implied herd behavior in stock markets
}

\author{
Daniël Linders* $\quad$ Jan Dhaene ${ }^{\dagger} \quad$ Wim Schoutens ${ }^{\ddagger}$
}

Version: December 19, 2014

\begin{abstract}
In this paper, we introduce two classes of indices which can be used to measure the market perception concerning the degree of dependency that exists between a set of random variables, representing different stock prices at a fixed future date. The construction of these measures is based on the theory of comonotonicity. Both types of herd behavior indices are model-free and risk-neutral, derived from available option data. Depending on its particular definition, each index represents a particular aspect of the market sentiment concerning future co-movement of the underlying stock prices.
\end{abstract}

Keywords: comonotonicity, herd behavior, HIX, index options, market fear, model-free measures, VIX.

\section{Introduction}

Never put all your eggs in one basket. Most investors are well aware of this advice and often prefer to invest in a blend of different stocks. This is generally considered as a prudent and risk-reducing strategy because losses caused by some of the assets may be countered by gains caused by others. However, periods of high market stress are typically linked to high levels of co-movement, implying that the diversification benefit is evaporating when it is needed most. Having a notion about today's level of co-movement (which we will also call the degree of herd behavior) may give market participants the opportunity to take necessary cautionary actions.

One possible way to investigate the degree of herd behavior between stock prices is to consider the historical performance of the multivariate time series of observed prices; see e.g. Kleykamp and Liu (2012), Harmon et al. (2011) and Bekaert et al. (2009). However,

*KU Leuven, Leuven, Belgium. Email: Daniel.Linders@econ.kuleuven.be

${ }^{\dagger}$ KU Leuven, Leuven, Belgium. Email: Jan.Dhaene@econ.kuleuven.be

${ }^{\ddagger}$ KU Leuven, Leuven, Belgium. Email: wim@schoutens.be 
as the dynamics of the co-movement between stock prices is changing over time, estimating future herd behavior based on historical data is a hard, if not impossible task.

In this paper, we take a prospective view and use currently observed option prices to gain insight in the level of herd behavior in the market over a given time interval. The prices of publicly traded options on an index (such as the Dow Jones Index) and on its underlying stocks contain information about the market's perception of future price co-movements. As such, an appropriately defined estimate of the degree of herd behavior based on such a set of observed option prices may reveal information about the view of the market concerning future co-movement. An implied degree of herd behavior that can be determined in a fast and robust way will allow us to monitor the evolution of the market perception concerning herd behavior in real time.

The CBOE S\&P 500 Implied Correlation Index is a quoted measure for the implied degree of co-movement, see Chicago Board Options Exchange (2009). A similar correlation measure for the Dow Jones is studied in Skintzi and Refenes (2005). Linders and Schoutens (2014b) propose a framework for the robust measurement of implied correlation levels between stocks composing a stock market index. The above-mentioned correlation measures can be determined from available option data. A drawback of these measures is that they are not model-free, as they are built on the assumption that the dynamics of stock prices is described by a multivariate lognormal distribution. It is well-known that this Gaussian assumption is often not an adequate approach to describe multivariate stock price dynamics. Moreover, all these measures are based on pairwise correlations, which may fail to capture the degree of herd behavior and could even give misleading signals; see e.g. Embrechts et al. (1999), Dhaene, Linders, Schoutens and Vyncke (2012) and Linders and Schoutens $(2014 b)$. A study of implied correlation for stock price models with non-normal marginal logreturns was conducted in Fonseca et al. (2007), Garcia et al. (2009), Tavin (2013), Austing (2014) and Linders and Schoutens (2014a).

In this paper, we will follow another road and use the concept of comonotonicity to define alternative measures for the implied degree of co-movement between stock prices. Roughly stated, the random variables (r.v.'s) representing the different stock prices in one month from now are said to be comonotonic in case they move in unison and behave like a single asset, not allowing for any diversification. In reality, stock prices will typically not move in a perfect comonotonic way and as such, a stock market is not exhibiting perfect herd behavior. Nevertheless, it is possible to construct the hypothetical comonotonic market situation out of the available stock option data. This artificial comonotonic market situation is then used as a point of reference, allowing us to measure the 'distance' between the real (observed) market situation and the comonotonic (non-observed) extreme case.

In Hobson et al. (2005) and Chen et al. (2008), it is explained how to determine index option prices corresponding to the extreme comonotonic market situation, from the prices of options written on the components of this stock market index. The observed index option prices are constrained from above by the (artificially constructed) comonotonic index option prices. Moreover, the gap between the observed index option curve, representing the real market situation, and the comonotonic index option curve, representing a market with perfect herd behavior, reveals information about the market perception 
concerning the strength of the co-movement present in the market. This gap is called the comonotonicity gap. A small gap might be interpreted as a sign for an almost comonotonic market situation where all stocks are moving together and almost no diversification is possible. A large gap, on the other hand, indicates that a high degree of diversification is possible. The terminology comonotonicity gap was first introduced in Laurence (2008). Dependence measures based on the comonoticity gap in a general setting, not related to option pricing, are investigated in Koch and De Schepper (2011) and Dhaene et al. (2014).

We propose a new methodology for capturing the comonotonicity gap in stock markets in a single and intuitive real number, called a herd behavior measure. First, we use the observed values of the stock market index (also called index) options expiring at a fixed date to construct an estimate for the swap rate with floating leg a convex function of the index value paid at that same date. The VIX formula, see Chicago Board Options Exchange (2003) and Carr and Wu (2006), turns out to be a special case of this general setting. In a second step, we determine the comonotonic index option curve, which represents the situation where all stocks in the index move perfectly together. The swap rate in the comonotonic situation is an upper bound for the real swap rate. We define the comonotonicity gap as the ratio between the swap rate and its comonotonic upper bound. Any convex function can be used to construct a model-free measure for the implied degree of co-movement, implying a whole class of herd behavior measures. The Herd Behavior Index (HIX) defined in Dhaene, Linders, Schoutens and Vyncke $(2012)$ and the Comonotonicity Index (CIX), defined in Dhaene, Dony, Forys, Linders and Schoutens (2012), turn out to be particular members of this class.

A second way of measuring herd behavior in stock markets that is presented in this paper is based on the concept of distorted expectations. We first show how to determine the distorted expectation of the stock index from the observed index option prices. Next, we determine the distorted expectation corresponding to the comonotonic situation. A second class of herd behavior measures is then defined by comparing the distorted expectation of the stock market index with its comonotonic modification. Each concave distortion function can be used to construct a model-free measure for the implied level of co-movement. Notice that the members of this second class of herd behavior measures can be considered as model-free counterparts of the Sector Diversity Index (SDI) defined in Madan and Schoutens (2013).

This paper is organized as follows. In Section 2 we present convex order and its connection with upper and lower tail transforms, as well as the notion of comonotonicity. The financial market we assume throughout the paper is introduced in Section 3. Section 4 describes how to determine the comonotonic market situation from observed stock options. We also introduce the comonotonicity gap and illustrate that during periods of increased market fear, this gap is tightening. The classes of herd behavior measures based on swap rates and on distorted expectations are defined in Section 5 and in Section 6, respectively. Section 7 concludes the paper. 


\section{Tail transforms, convex order and comonotonicity}

In this section, we present several concepts and results that will be used throughout the paper.

\section{$2.1 \quad$ Tail transforms}

The inverse of the cumulative distribution function (cdf) of a random variable (r.v.) $X$ is denoted by $F_{X}^{-1}$. It is defined by

$$
F_{X}^{-1}(p)=\inf \left\{x \in \mathbb{R} \mid F_{X}(x) \geq p\right\}, \quad p \in[0,1],
$$

with $\inf \emptyset=+\infty$, by convention. An alternative inverse of $F_{X}$ is defined as follows:

$$
F_{X}^{-1+}(p)=\sup \left\{x \in \mathbb{R} \mid F_{X}(x) \leq p\right\}, \quad p \in[0,1],
$$

where $\sup \emptyset=-\infty$, by convention. The inverses $F_{X}^{-1}(p)$ and $F_{X}^{-1+}(p)$ only differ on horizontal segments of the distribution function $F_{X}$. Notice that

$$
F_{X}^{-1}(p)=-F_{-X}^{-1+}(1-p)
$$

For any number $\alpha \in[0,1]$, the alpha inverse $F_{X}^{-1(\alpha)}$ is defined as a linear combination of the inverses (1) and (2):

$$
F_{X}^{-1(\alpha)}(p)=\alpha F_{X}^{-1}(p)+(1-\alpha) F_{X}^{-1+}(p), \quad p \in(0,1) .
$$

All r.v.'s that will be considered in this paper are assumed to have a finite mean. The Tail Value-at-Risk at level $p$, notation $\mathrm{TVaR}_{p}[X]$, of a r.v. $X$ is defined as follows:

$$
\mathrm{TVaR}_{p}[X]=\frac{1}{1-p} \int_{p}^{1} F_{X}^{-1}(q) \mathrm{d} q, \quad p \in(0,1) .
$$

$\mathrm{TVaR}_{p}$ can be considered as a measure for the upper tail of the cdf $F_{X}$ of $X$. It is linked with the upper tail transform $\mathbb{E}\left[(X-K)_{+}\right]$for an appropriate choice of $K$ :

$$
\operatorname{TVaR}_{p}[X]=F_{X}^{-1(\alpha)}(p)+\frac{1}{1-p} \mathbb{E}\left[\left(X-F_{X}^{-1(\alpha)}(p)\right)_{+}\right], \quad \alpha \in[0,1] \text { and } p \in(0,1)
$$

see e.g. Dhaene et al. (2006).

The Left Tail Value-at-Risk at level $p$ of $X$ is denoted by $\operatorname{LTVaR}_{p}[X]$. It is defined by

$$
\operatorname{LTVaR}_{p}[X]=\frac{1}{p} \int_{0}^{p} F_{X}^{-1}(q) \mathrm{d} q, \quad p \in(0,1) .
$$

$\operatorname{LTVaR}_{p}$ can be considered as a measure for the lower tail of the cdf $F_{X}$. Notice that

$$
\operatorname{LTVaR}_{p}[X]=-\mathrm{TVaR}_{1-p}[-X]
$$


Taking into account (3), (6) and (8), we find the following expression which relates $\operatorname{LTVaR}_{p}[X]$ with the lower tail transform $\mathbb{E}\left[(K-X)_{+}\right]$for an appropriate choice of $K$ :

$$
\operatorname{LTVaR}_{p}[X]=F_{X}^{-1(\alpha)}(p)-\frac{1}{p} \mathbb{E}\left[\left(F_{X}^{-1(\alpha)}(p)-X\right)_{+}\right], \quad \alpha \in[0,1] \text { and } p \in(0,1)
$$

Finally, notice that $\operatorname{TVaR}_{p}[X]$ and $\operatorname{LTVaR}_{p}[X]$ are connected by the following expression:

$$
p \operatorname{LTVaR}_{p}[X]+(1-p) \operatorname{TVaR}_{p}[X]=\mathbb{E}[X], \quad p \in(0,1) .
$$

\subsection{Convex order and comonotonicity}

The variability of (the cdf of) two r.v.'s can be compared via the notion of convex order; see e.g. Shaked and Shanthikumar (2007) or Denuit et al. (2005). Recall that convex order between two r.v.'s $X$ and $Y$, notation $X \preceq_{c x} Y$, is defined by

$$
X \preceq_{c x} Y \Leftrightarrow \begin{cases}\mathbb{E}\left[(X-K)_{+}\right] \leq \mathbb{E}\left[(Y-K)_{+}\right], & \text {for all } K \in \mathbb{R}, \\ \mathbb{E}\left[(K-X)_{+}\right] \leq \mathbb{E}\left[(K-Y)_{+}\right], & \text {for all } K \in \mathbb{R} .\end{cases}
$$

Intuitively, relation (11) indicates that $Y$ has larger lower and upper tails than $X$. This means that $X \preceq_{c x} Y$ can indeed be interpreted as ' $Y$ is more variable than $X$ '.

Two r.v.'s $X$ and $Y$ can only be ordered in convex order in case $\mathbb{E}[X]=\mathbb{E}[Y]$. Moreover, it is straightforward to prove that

$$
X \preceq_{c x} Y \Leftrightarrow\left\{\begin{array}{l}
\mathbb{E}\left[(X-K)_{+}\right] \leq \mathbb{E}\left[(Y-K)_{+}\right], \quad \text { for all } K \in \mathbb{R}, \\
\mathbb{E}[X]=\mathbb{E}[Y] .
\end{array}\right.
$$

Convex order can also be characterized in terms of TVaR's and LTVaR's:

$$
X \preceq_{c x} Y \Leftrightarrow\left\{\begin{array}{l}
\operatorname{TVaR}_{p}[X] \leq \operatorname{TVaR}_{p}[Y], \quad \text { for all } p \in(0,1) \\
\operatorname{LTVaR}_{p}[Y] \leq \operatorname{LTVaR}_{p}[X], \quad \text { for all } p \in(0,1) .
\end{array}\right.
$$

Consider a random vector $\underline{X}=\left(X_{1}, \ldots, X_{n}\right)$ with marginal distributions denoted by $F_{X_{i}}, i=1,2, \ldots, n$. The comonotonic modification of $\underline{X}$, notation $\underline{X}^{c}$, is defined by

$$
\underline{X}^{c} \stackrel{d}{=}\left(F_{X_{1}}^{-1}(U), \ldots, F_{X_{n}}^{-1}(U)\right)
$$

where $\stackrel{d}{=}$ stands for equality in distribution and $U$ is a r.v. which is uniformly distributed over the unit interval. The components of the random vector $\underline{X}^{c}$ are said to be comonotonic. Characterization (14) shows that a random vector is comonotonic if its components are jointly driven up or down by a single stochastic risk factor. Therefore, we say that $\underline{X}^{c}$ exhibits perfect herd behavior. For an introduction to the theory of comonotonicity, we refer to Dhaene et al. (2002a). Financial and actuarial applications are described in Dhaene et al. (2002b). An updated overview of applications of comonotonicity can be found in Deelstra et al. (2011). 
Let us introduce the notations

$$
S=w_{1} X_{1}+w_{2} X_{2}+\ldots+w_{n} X_{n}
$$

and

$$
S^{c}=w_{1} F_{X_{1}}^{-1}(U)+w_{2} F_{X_{2}}^{-1}(U)+\ldots+w_{n} F_{X_{n}}^{-1}(U),
$$

for the weighted sums of the components of $\underline{X}$ and $\underline{X}^{c}$, with deterministic positive weight factors $w_{i}$. It can be proven that

$$
S \leq_{c x} S^{c}
$$

which means that in the class of all random vectors with given marginal distributions, a comonotonic situation leads to a weighted sum which is largest in convex order.

\section{The stock market}

\subsection{Stocks, the stock market index and a finite market of options}

We consider a financial market ${ }^{1}$ where $n$ (dividend or non-dividend paying) stocks, labeled from 1 to $n$, are traded. Suppose that the current time is $t=0$. The price at time $t$, $0 \leq t \leq T<+\infty$, of stock $i$ is denoted by $X_{i}(t)$. Unless otherwise stated, we will always silently assume that $X_{i}(t) \geq 0$ for all $i$ and that its first and second order moments are finite. Apart from the stocks, there is a stock market index of which the price is a linear combination of the prices of the $n$ underlying stocks. Denoting the price of the index at time $t$ by $S(t), 0 \leq t \leq T$, we have that

$$
S(t)=w_{1} X_{1}(t)+w_{2} X_{2}(t)+\ldots+w_{n} X_{n}(t),
$$

where $w_{i}, i=1,2, \ldots, n$, are positive weights that are fixed up front.

The time-0 prices of the European-type index call and put options with strike $K$ and maturity $T$ are denoted by $C[K, T]$ and $P[K, T]$, respectively. Further, the time- 0 prices of European-type calls and puts on stock $i$ are denoted by $C_{i}[K, T]$ and $P_{i}[K, T]$, respectively.

The financial market is assumed to be arbitrage-free and there exists a pricing measure $\mathbb{Q}$, equivalent to the physical probability measure $\mathbb{P}$, such that the current price of any pay-off at time $T$ can be represented as the expectation of the discounted pay-off. In this price-recipe, the discounting factor is $\mathrm{e}^{-r T}$, where $r$ is the continuously compounded time0 risk-free interest rate, while expectations are taken with respect to the pricing measure $\mathbb{Q}$. For simplicity reasons, we assume deterministic interest rates. Notice however that all results hereafter remain to hold in case interest rates are stochastic, provided the discounting factor $\mathrm{e}^{-r T}$ is interpreted as the time-0 price of a $T$-year zero coupon bond

\footnotetext{
${ }^{1}$ We use the common approach to describe the financial market via a filtered probability space $\left(\Omega, \mathcal{F},\left(\mathcal{F}_{t}\right)_{0 \leq t \leq T}, \mathbb{P}\right)$.
} 
and the pricing measure $\mathbb{Q}$ is interpreted as a ' $T$-year forward measure' instead of a 'risk-neutral measure', see Chen et al. (2015).

The no-arbitrage condition gives rise to the following expressions for the option prices:

$$
\begin{aligned}
C_{i}[K, T] & =\mathrm{e}^{-r T} \mathbb{E}\left[\left(X_{i}(T)-K\right)_{+}\right], \\
P_{i}[K, T] & =\mathrm{e}^{-r T} \mathbb{E}\left[\left(K-X_{i}(T)\right)_{+}\right],
\end{aligned}
$$

and

$$
\begin{aligned}
& C[K, T]=\mathrm{e}^{-r T} \mathbb{E}\left[(S(T)-K)_{+}\right], \\
& P[K, T]=\mathrm{e}^{-r T} \mathbb{E}\left[(K-S(T))_{+}\right] .
\end{aligned}
$$

In formulae (19), (20) and (21), (22), as well as in the remainder of this text, expectations (distributions) of functions of $\left(X_{1}(T), \ldots, X_{n}(T)\right)$ have to be understood as expectations (distributions) under the $\mathbb{Q}$-measure. Furthermore, the notations $F_{X_{i}(T)}$ and $F_{S(T)}$ will be used for the time-0 cdf's of $X_{i}(T)$ and $S(T)$ under the measure $\mathbb{Q}$.

In order to avoid unnecessary overloading of the notations, hereafter we will omit the fixed time index $T$ when no confusion is possible. For example, we will write $X_{i}, C_{i}[K]$ and $F_{X_{i}}(x)$ for $X_{i}(T), C_{i}[K, T]$ and $F_{X_{i}(T)}(x)$, respectively.

Throughout this paper, we will assume that market participants have only access to a finite number of European-type options with maturity $T$. In particular, for any stock $i$, the strikes of the traded puts and calls are denoted by $K_{i, j}, j=-l_{i},-l_{i}+1, \ldots, h_{i}-1, h_{i}$, with

$$
0=K_{i,-l_{i}}<\ldots<K_{i,-1}<K_{i, 0} \leq \mathbb{E}\left[X_{i}\right]<K_{i, 1}<K_{i, 2}<\ldots<K_{i, h_{i}}<K_{i, h_{i}+1},
$$

where $K_{i, h_{i}+1}=F_{X_{i}}^{-1}(1)$ is assumed to be finite. In reality, stock and call options may have an unknown upward potential. However, for numerical reasons, we will enforce a finite upper bound which can be chosen arbitrarily large. The strikes of the traded puts and calls on the stock index are denoted by $K_{j}, j=-l, \ldots, h$, with

$$
0 \leq K_{-l}<K_{-l+1}<\ldots<K_{0} \leq \mathbb{E}[S]<K_{1}<K_{2}<\ldots<K_{h}<K_{h+1},
$$

where $K_{h+1}=F_{S}^{-1}(1)$. Note that as long as there is at least one strike $K$ for which the prices $C[K]$ and $P[K]$ are traded, the forward rate $\mathbb{E}[S]$ can be calculated in a model-free way using the put-call parity. In practical situations, we follow the methodology proposed in Chicago Board Options Exchange (2003) to determine the forward rate of the stock market index:

$$
\mathbb{E}[S]=\mathrm{e}^{r T}\left(C\left[K^{*}\right]-P\left[K^{*}\right]\right)+K^{*}
$$

with

$$
K^{*}=\arg \min _{K \in\left\{K_{-l}, \ldots K_{h_{-} 1}\right\}}|C[K]-P[K]| .
$$

For more details, see also Linders et al. (2012). A similar approach is followed for determining the forward rate of the individual stocks. 


\subsection{Index option prices and convex order}

Consider two points in time, $t$ and $t^{\prime}$, with $0 \leq t \leq t^{\prime}$. Let $S$ and $S^{\prime}$ be the r.v.'s representing the time $(t+T)$ - and time $\left(t^{\prime}+T\right)$ - index prices and $\mathbb{E}[S]$ and $\mathbb{E}^{\prime}\left[S^{\prime}\right]$ the $T$-year forward prices, seen from the viewpoint of time $t$ and time $t^{\prime}$, respectively. The corresponding forward deflated time $(t+T)$ - and time $\left(t^{\prime}+T\right)$ - index prices are defined by $\frac{S}{\mathbb{E}[S]}$ and $\frac{S^{\prime}}{\mathbb{E}^{\prime}\left[S^{\prime}\right]}$, while the respective European-type call options on the index, with time-to-maturity $T$ and strike $K$, are denoted by $C[K]$ and $C^{\prime}[K]$. From (12) it follows that

$$
\frac{S}{\mathbb{E}[S]} \preceq_{c x} \frac{S^{\prime}}{\mathbb{E}^{\prime}\left[S^{\prime}\right]} \Leftrightarrow \frac{C[k \mathbb{E}[S]]}{\mathbb{E}[S]} \leq \frac{C^{\prime}\left[k \mathbb{E}^{\prime}\left[S^{\prime}\right]\right]}{\mathbb{E}^{\prime}\left[S^{\prime}\right]}, \quad \text { for all } k \geq 0 .
$$

The non-negative real number $k$ in the right-hand side inequality in (27) is called the moneyness of the option under consideration. From (27), it follows that, having arrived at time $t^{\prime}$, one can search for eventual convex order relations between deflated index prices

$\frac{S}{\mathbb{E}[S]}$ and $\frac{S^{\prime}}{\mathbb{E}\left[S^{\prime}\right]}$ by comparing the corresponding $T$-year index option curves. An eventual convex order will indicate an increased degree of variabilty of the $T$-year stock index price at time $t^{\prime}$, compared to time $t$. Such an increased level of index variability can be caused by an increased level of variability of the individual stocks, an increased co-movement between the individual stocks or a combination of these two effects. A related study was conducted in Carr et al. (2011), where the authors consider the monotonicity in convex order by comparing options on standardized realized variance for different maturities.

In order to illustrate this procedure, let us look for eventual convex order relations between deflated Dow Jones index prices. Recall that the Dow Jones Industrial Average is a price-weighted index composed of the 30 largest, most liquid NYSE and NASDAQ listed stocks. Options with the DJ index as underlying are called DJX options and are based on $1 / 100$ th of the current value of the DJ.

Figure 1 shows the values $\frac{C[k \mathbb{E}[S]]}{\mathbb{E}[S]}$ (closing mid-prices) for the traded options with time-to-maturity $T=30$ days, for trading days April 17, 2008 (dots), October 23, 2008 (crosses), November 20, 2008 (circles) and December 18, 2008 (plusses). From Figure 1, we can conclude that during the period October - December 2008, the deflated index prices are larger in convex order than during April 2008. Furthermore, the month November 2008 was the most extreme one, in terms of convex order.

\section{Perfect herd behavior and the comonotonicity gap}

\subsection{Comonotonic index option prices}

In this section, we show how to construct a synthetic $T$-year stock market index price with a risk-neutral cdf which is maximal in convex order, in the class of all risk-neutral distributions for the $T$-year stock market index price which are consistent with the observed stock option data.

Recall that we assumed that for each stock option curve $C_{i}$, only the points $\left(K_{i, j}, C_{i}\left[K_{i, j}\right]\right)$, $j=-l_{i}, \ldots, h_{i}+1$, are observed, see (23). Following Hobson et al. (2005) and Chen et al. 


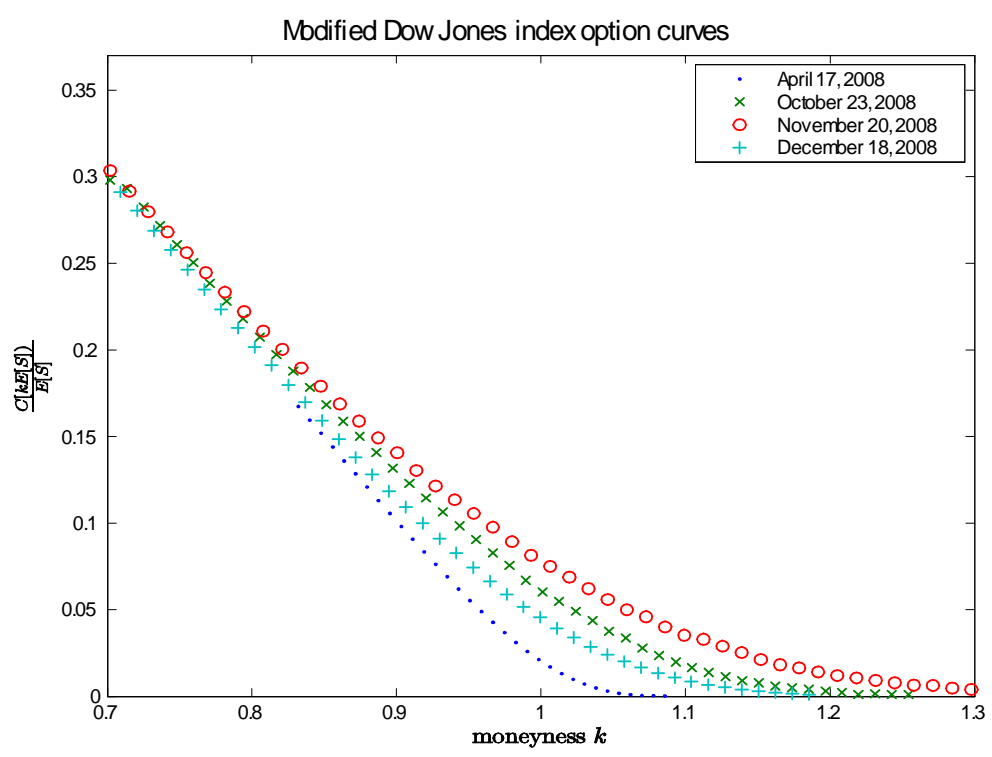

Figure 1: The index option curve $\frac{C[k \mathbb{E}[S]]}{\mathbb{E}[S]}$ for different moneyness values $k$.

(2008), we approximate the partially known convex call option curve $C_{i}$ by the piecewise linear convex curve $\bar{C}_{i}$ which connects its observed points.

Let us introduce the notation $\bar{X}_{i}$ for a r.v. with cdf equal to the cdf implied by the option curve $\bar{C}_{i}$. This cdf $F_{\bar{X}_{i}}$ can be considered as an approximation for the unknown risk-neutral pricing cdf $F_{X_{i}}$. It can be determined from the observed stock option prices via

$$
F_{\bar{X}_{i}}(x)=1+\mathrm{e}^{r T} \bar{C}_{i}^{\prime}[x+]
$$

where, $\bar{C}_{i}^{\prime}[x+]$ is the right derivative of $\bar{C}_{i}$ at $x$, see e.g. Breeden and Litzenberger (1978). Notice that using the put-call parity, expression (28) can be recast in a relation for the risk-neutral cdf $F_{\bar{X}_{i}}$ in terms of the observed put option prices.

One can easily verify that the $\operatorname{cdf} F_{\bar{X}_{i}}$ defined above, is given by

$$
F_{\bar{X}_{i}}(x)=\left\{\begin{array}{cc}
0 & \text { if } x<0, \\
1+\mathrm{e}^{r T} \frac{C_{i}\left[K_{i, j+1}\right]-C_{i}\left[K_{i, j}\right]}{K_{i, j+1}-K_{i, j}} & \text { if } K_{i, j} \leq x<K_{i, j+1}, \\
1 & \left(j=-l_{i}, \ldots, h_{i}+1\right) \\
& \text { if } x \geq K_{i, h_{i}+1} .
\end{array}\right.
$$

In the sequel, we will always assume that $F_{\bar{X}_{i}}$ is strictly increasing in the traded strikes whenever the cdf is strictly positive in this strike price, in the following sense: if $F_{\bar{X}_{i}}\left(K_{i, j}\right)>0$, then $F_{\bar{X}_{i}}\left(K_{i, j}\right)>F_{\bar{X}_{i}}\left(K_{i, j-1}\right)$ for $j=-l_{i}, \ldots, h_{i}+1$, with $K_{i,-l_{i}-1}=-1$ by convention. If this condition is initially not satisfied, we decrease the set of observed points until it is satisfied. From (29) it follows then that for any $j=-l_{i}, \ldots, h_{i}+1$ and $q \in(0,1]$, the inverse $F_{\bar{X}_{i}}^{-1}$ is determined by

$$
F_{\bar{X}_{i}}^{-1}(q)=K_{i, j}, \quad \text { if } F_{\bar{X}_{i}}\left(K_{i, j-1}\right)<q \leq F_{\bar{X}_{i}}\left(K_{i, j}\right) .
$$


Next, we introduce the r.v. $\bar{S}^{c}$, which is defined by

$$
\bar{S}^{c}=F_{\bar{X}_{1}}^{-1}(U)+F_{\bar{X}_{2}}^{-1}(U)+\ldots+F_{\bar{X}_{n}}^{-1}(U) .
$$

We will call $\bar{S}^{c}$ the $T$-year comonotonic stock market index price. Its cdf can be determined from the marginal cdf's $F_{\bar{X}_{i}}$. The comonotonic call and put option prices $\bar{C}^{c}[K]$ and $\bar{P}^{c}[K]$ are defined by

$$
\bar{C}^{c}[K]=\mathrm{e}^{-r T} \mathbb{E}\left[\left(\bar{S}^{c}-K\right)_{+}\right]
$$

and

$$
\bar{P}^{c}[K]=\mathrm{e}^{-r T} \mathbb{E}\left[\left(K-\bar{S}^{c}\right)_{+}\right]
$$

respectively.

The outcome of the comonotonic stock market index $\bar{S}^{c}$ will never be observed. However, in the hypothetical case that the stock option curves are piecewise linear and moreover, the time- $T$ stock prices are comonotonic, the risk-neutral distribution of the time$T$ index price will be equal to the distribution of $\bar{S}^{c}$. In this case, it turns out that $C[K]=\bar{C}^{c}[K]$ and $P[K]=\bar{P}^{c}[K]$ for all strikes $K$. Notice that comonotonicity is maintained under equivalent probability measures. The reachability of the comonotonic market situation in the case of finite stock option data is considered in Hobson et al. (2005).

Obviously, in practice the pricing measure will never be such that the index option curves coincide with $\bar{C}^{c}[K]$ and $\bar{P}^{c}[K]$. However, the convex order relation

$$
S \leq_{c x} \bar{S}^{c}
$$

a proof of which can be found in Chen et al. (2008), together with the convex order characterization (11) implies that

$$
C[K] \leq \bar{C}^{c}[K]
$$

and

$$
P[K] \leq \bar{P}^{c}[K]
$$

hold for any strike $K \geq 0$. This means that the comonotonic index option prices $\bar{C}^{c}[K]$ and $\bar{P}^{c}[K]$ are model-free upper bounds for the index option prices $C[K]$ and $P[K]$, respectively.

The inequality (34) shows that for a given finite set of stock option prices, the comonotonic stock market index $\bar{S}^{c}$ describes the extreme situation corresponding to a maximal level of variability, expressed in terms of convex order. Indeed, any other feasible market situation which is consistent with the observed stock option data will result in an index $S$ which will not exceed $\bar{S}^{c}$ in convex order. The maximal level of volatility is attained when the market is pricing (multi-asset) derivatives using the cdf of the comonotonic random vector $\left(F_{\bar{X}_{1}}^{-1}(U), F_{\bar{X}_{2}}^{-1}(U), \ldots, F_{\bar{X}_{n}}^{-1}(U)\right)$. 
In the following theorem, the comonotonic call option prices $\bar{C}^{c}[K]$ and $\bar{P}^{c}[K]$ are expressed in terms of observed stock option prices. Similar results can be derived for comonotonic put option prices. A proof of this theorem can be found in Hobson, Laurence and Wang (2005). A simplified proof of this result, based on the decomposition formula for stop-loss premiums of a comonotonic sum (see Dhaene et al. (2000)), can be found in Chen et al. (2008).

Theorem 1 For any $K \in\left(F_{\bar{S}^{c}}^{-1+}(0), F_{\bar{S}^{c}}^{-1}(1)\right)$, the comonotonic index option prices $\bar{C}^{c}[K]$ and $\bar{P}^{c}[K]$ can be expressed as

$$
\begin{aligned}
& \bar{C}^{c}[K]=\sum_{i=1}^{n} w_{i} \bar{C}_{i}\left[K_{i}^{*}\right], \\
& \bar{P}^{c}[K]=\sum_{i=1}^{n} w_{i} \bar{P}_{i}\left[K_{i}^{*}\right],
\end{aligned}
$$

with the strikes $K_{i}^{*}$ given by

$$
K_{i}^{*}=F_{\bar{X}_{i}}^{-1\left(\alpha_{K}\right)}\left(F_{\bar{S}^{c}}(K)\right), \quad i=1,2, \ldots, n
$$

and where $\alpha_{K}$ is any element in $[0,1]$ satisfying

$$
F_{\bar{S}^{c}}^{-1\left(\alpha_{K}\right)}\left(F_{\bar{S}^{c}}(K)\right)=K \text {. }
$$

When $K \notin\left(F_{\bar{S}^{c}}^{-1+}(0), F_{\bar{S}^{c}}^{-1}(1)\right)$, the comonotonic prices $\bar{C}^{c}[K]$ and $\bar{P}^{c}[K]$ can be determined in a straightforward way and coincide with the prices $C[K]$ and $P[K]$, respectively. A detailed and robust step-by-step algorithm to determine the upper bounds $\bar{C}^{c}[K]$ and $\bar{P}^{c}[K]$ in an efficient way can be found in Linders et al. (2012).

In the above-mentioned paper, several known optimality results of the upper bounds $\bar{C}^{c}[K]$ and $\bar{P}^{c}[K]$ are summarized. In particular, it is shown that $\bar{C}^{c}[K]$ and $\bar{P}^{c}[K]$ are the least upper bounds for the respective index option prices, in the class of all pricing models which are consistent with the observed stock option prices.

\subsection{The comonotonicity gap}

Laurence (2008) introduced the term comonotonicity gap to indicate the ratio between the observed market price $C[K]$, resp. $P[K]$, and the comonotonic index option price $\bar{C}^{c}[K]$, resp. $\bar{P}^{c}[K]$, for an appropriate choice of $K$. Obviously, the $T$-year comonotonicity gap varies over time. A small gap indicates that the distance between the real market situation and the theoretical comonotonic situation is small, which can be interpreted as a signal for a market driven by the perception that stocks will move strongly together over the next $T$ years. 
Figure 2 shows out-of-the-money Dow Jones index option prices and their comonotonic upper bounds on different trading days for the set of traded strikes. To be more precise, for a moneyness $k<1$, we compare the index put option prices $P[k \mathbb{E}[S]]$ with their respective comonotonic modifications $\bar{P}^{c}[k \mathbb{E}[S]]$, whereas $C[k \mathbb{E}[S]]$ and $\bar{C}^{c}[k \mathbb{E}[S]]$ are used when $k \geq 1$. Hence, the options involved are all out-of-the money options, which are in general more liquid than in-the-money options. Moreover, the prices of out-of-the money options contain only time value and no intrinsic value. The options expire in 30 days. As before, the trading days under consideration are April 17, 2008, October 23, 2008, November 20, 2008 and December 18, 2008. From this figure, one observes that the distance between the two option curves as well as their height is changing over time, indicating a changing degree of implied herd behavior.

The at-the-money comonotonicity gap is defined as

$$
\text { ATM comonotonicity gap }=\frac{C\left[K_{0}\right]}{\bar{C}^{c}\left[K_{0}\right]},
$$

where $K_{0}$ is the largest traded strike not exceeding the index forward price $\mathbb{E}[S]$. On April 17, 2008, the at-the-money comonotonicity gap is $61.09 \%$. The period October November 2008 is in the heat of the financial crisis, where obviously a high degree of herd behavior was observed. In this period, the at-the-money comonotonicity gap is $82.90 \%$ and 80.39\% for October 23, 2008 and November 20, 2008, respectively. In December 18, 2008, we observe a comonotonicity gap of $75.58 \%$; see Table 1 .

The ratio $\frac{\bar{Q}^{c}[k \mathbb{E}[S]]}{Q[k \mathbb{E}[S]]}$ for the traded strikes is shown in Figure 3 , where

$$
Q[K]=\left\{\begin{array}{ll}
P[K], & \text { if } K<\mathbb{E}[S], \\
C[K], & \text { if } K \geq \mathbb{E}[S],
\end{array} \quad \text { and } \bar{Q}^{c}[K]= \begin{cases}\bar{P}^{c}[K], & \text { if } K<\mathbb{E}[S], \\
\bar{C}^{c}[K], & \text { if } K \geq \mathbb{E}[S]\end{cases}\right.
$$

Comparing Figures 2 and 3 shows that the conclusions drawn from these figures are similar. However, Figure 3 shows that November 2008 exhibits a lower degree of herd behavior than October 2008 when we consider out-of-the money index put options (i.e. when $k<1$ ), whereas the opposite relation holds when comparing out-of-the money index call options (when $k \geq 1$ ).

In Figure 1, we showed that we have the following convex order relation: April $08 \leq_{c x}$ December $08 \leq_{c x}$ October $08 \leq_{c x}$ November 08.

However, if we compare the degree of herd behavior (using the ATM comonotonicity gap) of these trading days, we find a different ordering:

$$
\text { April } 08 \leq_{h b} \text { October } 08 \leq_{h b} \text { November } 08 \leq_{h b} \text { December } 08 \text {. }
$$

where we use the notation $T_{1} \leq_{h b} T_{2}$ to indicate that the comonotonicity ratio is higher at time $T_{2}$ than at time $T_{1}$. The observed difference in the orderings is not necessarily a contradiction. Indeed, convex order gives information about the variability of the $T$-year index price, whereas the herd behavior ordering gives information about the distance towards comonotonicity. 

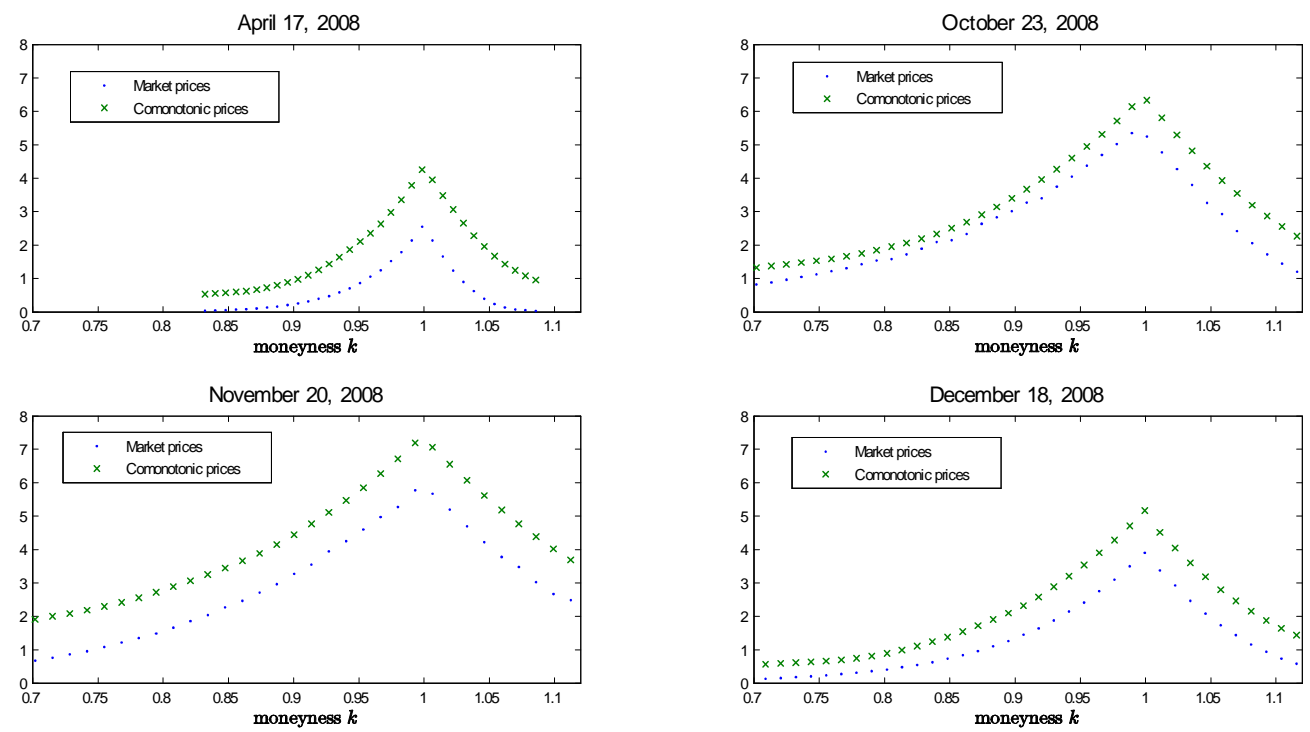

Figure 2: Out-of-the-money index option prices (dots) and comonotonic out-of-the-money index option prices (crosses) as a funtion of moneyness, for traded strikes.

In the following two sections, we propose two frameworks for quantifying the comonotonicity gap in a more sophisticated way than via the ATM comonotonicity gap. These frameworks are based on the idea that the relative distance of the observed index option curve from its comonotonic counterpart reveals information about the strength of the co-movement. Instead of comparing a particular index option with its comonotonic modification, we will use the full range of observed stock and index option prices to define two classes of implied indicators for the degree of herd behavior.

\section{Swap rates and implied herd behavior}

\subsection{Swap rates in terms of observed option prices}

Consider the swap contract of which the floating leg pays the buyer (i.e. the long party) the pay-off $u\left(\frac{S}{\mathbb{E}[S]}\right)$ at time $T$, for some convex function $u:[0,+\infty) \rightarrow \mathbb{R}$. In exchange, the fixed leg pays the seller the premium $P$ (also called the swap rate) at time $T$. The premium is agreed upon at the deal's inception and is set such that the contract has price 0 at time 0 :

$$
0=\mathrm{e}^{-r T} \mathbb{E}\left[u\left(\frac{S}{\mathbb{E}[S]}\right)-P\right]
$$

This swap contract amounts to a $T$-year forward contract written on a convex function $u$ of the forward deflated index price $\frac{S}{\mathbb{E}[S]}$. 


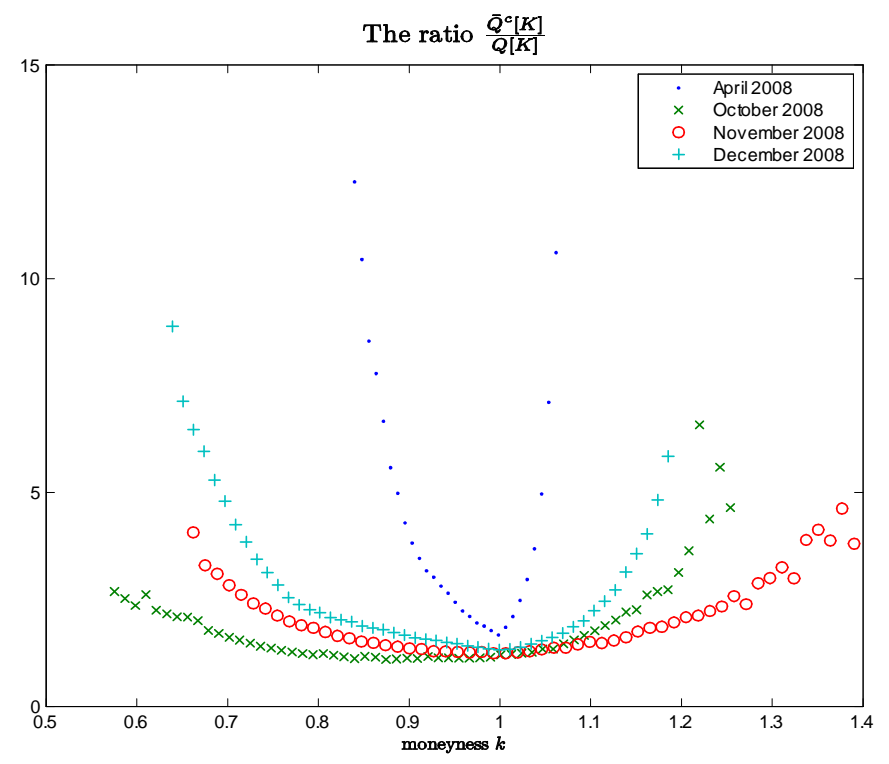

Figure 3: The ratios $\frac{\bar{Q}^{c}[k \mathbb{E}[S]]}{Q[k \mathbb{E}[S]]}$ as a function of the moneyness, for traded strikes.

Let us now assume that the derivative of $u$ (right derivative in 0 ) is well-defined and finite on $[0,+\infty)$, and that this derivative is absolutely continuous on $[0,+\infty)$. In this case, the swap rate $\mathbb{E}\left[u\left(\frac{S}{\mathbb{E}[S]}\right)\right]$ can be expressed as

$$
\begin{aligned}
& \mathbb{E}\left[u\left(\frac{S}{\mathbb{E}[S]}\right)\right]-u(1) \\
& \quad=\frac{\mathrm{e}^{r T}}{\mathbb{E}[S]^{2}}\left(\int_{0}^{\mathbb{E}[S]} u^{\prime \prime}\left(\frac{K}{\mathbb{E}[S]}\right) P[K] \mathrm{d} K+\int_{\mathbb{E}[S]}^{+\infty} u^{\prime \prime}\left(\frac{K}{\mathbb{E}[S]}\right) C[K] \mathrm{d} K\right),
\end{aligned}
$$

see e.g. Carr and Madan (2001) or Cheung et al. (2013). This is a model-free expression for the swap rate, in terms of prices of put options with strikes smaller than or equal to $\mathbb{E}[S]$ and call options with strikes larger than $\mathbb{E}[S]$. Notice that $\mathbb{E}[S]$ is usually relatively close to the current index price, implying that most options involved in (43) are out-of-the-money.

Our assumption that only a limited number of index options are traded, see (24), implies that the swap rate $\mathbb{E}\left[u\left(\frac{S}{\mathbb{E}[S]}\right)\right]$ cannot be determined from 43 . Inspired by the VIX methodology, we propose to approximate the integrals in (43), making use of the available index option data.

Let us first introduce the notations $\Delta K_{j}$, which are related to the strike grid:

$$
\Delta K_{j}=\left\{\begin{array}{cc}
K_{-l+1}-K_{-l}, & \text { if } j=-l, \\
\frac{K_{j+1}-K_{j-1}}{2}, & \text { if } j=-l+1, \ldots, h-1, \\
K_{h}-K_{h-1}, & \text { if } j=h .
\end{array}\right.
$$


Furthermore, we will use the notation $Q\left[K_{j}\right]$ which is defined by

$$
Q\left[K_{j}\right]=\left\{\begin{array}{cc}
P\left[K_{j}\right], & \text { if } K_{j}<K_{0}, \\
\frac{C\left[K_{j}\right]+P\left[K_{j}\right],}{2} & \text { if } K_{j}=K_{0}, \\
C\left[K_{j}\right], & \text { if } K_{j}>K_{0} .
\end{array}\right.
$$

Making use of the composite trapezoidal rule, we consider the following model-free approximation for the forward rate $\mathbb{E}\left[u\left(\frac{S}{\mathbb{E}[S]}\right)\right]$ in terms of observed index option prices:

$$
\mathbb{E}\left[u\left(\frac{S}{\mathbb{E}[S]}\right)\right]-u(1) \approx \frac{\mathrm{e}^{r T}}{\mathbb{E}[S]^{2}} \sum_{j=-l}^{h} u^{\prime \prime}\left(\frac{K_{j}}{\mathbb{E}[S]}\right) \Delta K_{j} Q\left[K_{j}\right]-\frac{u^{\prime \prime}\left(\frac{K_{0}}{\mathbb{E}[S]}\right)}{2}\left(\frac{\mathbb{E}[S]-K_{0}}{\mathbb{E}[S]}\right)^{2} .
$$

In order to show how we arrive at this approximation, we first rewrite the sum of the two integrals in (43) in the following way:

$$
\begin{aligned}
\int_{0}^{K_{0}} u^{\prime \prime}\left(\frac{K}{\mathbb{E}[S]}\right) P[K] \mathrm{d} K+\int_{K_{0}}^{+\infty} u^{\prime \prime} & \left(\frac{K}{\mathbb{E}[S]}\right) C[K] \mathrm{d} K \\
& +\int_{K_{0}}^{\mathbb{E}[S]} u^{\prime \prime}\left(\frac{K}{\mathbb{E}[S]}\right)(P[K]-C[K]) \mathrm{d} K .
\end{aligned}
$$

The first two integrals in this expression are then approximated using the composite trapezoidal rule. Assuming that $P[K]$ is equal to zero in $K_{-l}-\left(K_{-l+1}-K_{-l}\right)$, leads to the following approximation for the first integral:

$$
\int_{0}^{K_{0}} u^{\prime \prime}\left(\frac{K}{\mathbb{E}[S]}\right) P[K] \mathrm{d} K \approx \sum_{j=-l}^{-1} \Delta K_{i} u^{\prime \prime}\left(\frac{K_{j}}{\mathbb{E}[S]}\right) Q\left[K_{j}\right]+\frac{K_{0}-K_{-1}}{2} u^{\prime \prime}\left(\frac{K_{0}}{\mathbb{E}[S]}\right) P\left[K_{0}\right] .
$$

Assuming that $C[K]$ is equal to zero in $K_{h}+\left(K_{h}-K_{h-1}\right)$, we find a similar approximation for the second integral in (47):

$$
\int_{K_{0}}^{+\infty} u^{\prime \prime}\left(\frac{K}{\mathbb{E}[S]}\right) C[K] \mathrm{d} K \approx \frac{K_{1}-K_{0}}{2} u^{\prime \prime}\left(\frac{K_{0}}{\mathbb{E}[S]}\right) C\left[K_{0}\right]+\sum_{j=1}^{h} \Delta K_{j} u^{\prime \prime}\left(\frac{K_{j}}{\mathbb{E}[S]}\right) Q\left[K_{j}\right] .
$$

Furthermore, assuming that $u^{\prime \prime}\left(\frac{K}{\mathbb{E}[S]}\right)=u^{\prime \prime}\left(\frac{K_{0}}{\mathbb{E}[S]}\right)$ for $K \in\left[K_{0}, \mathbb{E}[S]\right]$ and taking into account the put-call parity leads to the following approximation for the third integral in (47):

$$
\int_{K_{0}}^{\mathbb{E}[S]} u^{\prime \prime}\left(\frac{K}{\mathbb{E}[S]}\right)(P[K]-C[K]) \mathrm{d} K \approx-\frac{e^{-r T}}{2} u^{\prime \prime}\left(\frac{K_{0}}{\mathbb{E}[S]}\right)\left(\mathbb{E}[S]-K_{0}\right)^{2} .
$$

Finally, assuming that $K_{1}-K_{0}=K_{0}-K_{-1}$ and taking into account (47), (48), (49) and (50), leads to the appproximation (46) for the swap rate.

The extra term $\frac{u^{\prime \prime}\left(\frac{K_{0}}{\mathbb{E}[S]}\right)}{2}\left(\frac{\mathbb{E}[S]-K_{0}}{\mathbb{E}[S]}\right)^{2}$ in 460 is a contribution due to the discretization around the forward rate $\mathbb{E}[S]$. Notice that in the approximation above, we silently assumed that all second derivatives $u^{\prime \prime}\left(\frac{K_{j}}{\mathbb{E}[S]}\right), j=-l, \ldots, h$, are well-defined and finite. 


\subsection{Swap rates in case of perfect herd behavior}

Let us now consider the comonotonic market situation. In this case, the swap rate is given by $\mathbb{E}\left[u\left(\frac{S^{c}}{\mathbb{E}[S]}\right)\right]$, with $S^{c}$ defined in 16 .

Due to our assumption about the finite number of traded stock options, see (24), we are not able to determine $\mathbb{E}\left[u\left(\frac{S^{c}}{\mathbb{E}[S]}\right)\right]$. Therefore, we propose to approximate it by $\mathbb{E}\left[u\left(\frac{\bar{S}^{c}}{\mathbb{E}[S]}\right)\right]$, with $\bar{S}^{c}$ defined in 31 . Similar to 43 , we find the following expression for the comonotonic swap rate:

$$
\begin{aligned}
& \mathbb{E}\left[u\left(\frac{\bar{S}^{c}}{\mathbb{E}[S]}\right)\right]-u(1) \\
& \quad=\frac{\mathrm{e}^{r T}}{\mathbb{E}[S]^{2}}\left(\int_{0}^{\mathbb{E}[S]} u^{\prime \prime}\left(\frac{K}{\mathbb{E}[S]}\right) \bar{P}^{c}[K] \mathrm{d} K+\int_{\mathbb{E}[S]}^{+\infty} u^{\prime \prime}\left(\frac{K}{\mathbb{E}[S]}\right) \bar{C}^{c}[K] \mathrm{d} K\right) .
\end{aligned}
$$

This comonotonic swap rate can be interpreted as the swap rate that would arise in a market with piecewise linear option curves and with comonotonic stock prices. Inspired by (46), we approximate (51) in the following way:

$\mathbb{E}\left[u\left(\frac{\bar{S}^{c}}{\mathbb{E}[S]}\right)\right]-u(1) \approx \frac{\mathrm{e}^{r T}}{\mathbb{E}[S]^{2}} \sum_{j=-l}^{h} u^{\prime \prime}\left(\frac{K_{j}}{\mathbb{E}[S]}\right) \Delta K_{j} \bar{Q}^{c}\left[K_{j}\right]-\frac{u^{\prime \prime}\left(\frac{K_{0}}{\mathbb{E}[S]}\right)}{2}\left(\frac{\mathbb{E}[S]-K_{0}}{\mathbb{E}[S]}\right)^{2}$.

In this expression, the $\Delta K_{j}$ are given by 44 , while the $\bar{Q}^{c}\left[K_{j}\right]$ are defined by

$$
\bar{Q}^{c}\left[K_{j}\right]=\left\{\begin{array}{cc}
\bar{P}^{c}\left[K_{j}\right], & \text { if } K_{j}<K_{0}, \\
\frac{\bar{C}^{c}\left[K_{j}\right]+\bar{P}^{c}\left[K_{j}\right]}{2}, & \text { if } K_{j}=K_{0}, \\
\bar{C}^{c}\left[K_{j}\right], & \text { if } K_{j}>K_{0} .
\end{array}\right.
$$

Although the comonotonic index option curves $\bar{C}^{c}$ and $\bar{P}^{c}$ are not observed, Theorem 1 allows us to determine the $\bar{Q}\left[K_{j}\right]$ from the available stock option data. We can conclude that $\left[52\right.$ is a model-free approximation for the comonotonic swap rate $\mathbb{E}\left[u\left(\frac{\bar{S}^{c}}{\mathbb{E}[S]}\right)\right]$ in terms of observed stock option prices.

\subsection{Measuring the degree of herd behavior in terms of swap rates}

Consider two points in time, $t$ and $t^{\prime}$, with $0 \leq t \leq t^{\prime}$. It is well known that for any convex function $u$, the following implication holds:

$$
\frac{S}{\mathbb{E}[S]} \leq_{c x} \frac{S^{\prime}}{\mathbb{E}^{\prime}\left[S^{\prime}\right]} \Longrightarrow \mathbb{E}\left[u\left(\frac{S}{\mathbb{E}[S]}\right)\right] \leq \mathbb{E}^{\prime}\left[u\left(\frac{S^{\prime}}{\mathbb{E}^{\prime}\left[S^{\prime}\right]}\right)\right]
$$


provided the expectations exist and where we use the notations and conventions introduced in (27). This means that convex order between deflated index price levels results in an ordering of the corresponding swap rates. For the special case of a convex $u$ with an absolute continuous derivative, this implication follows from (11) and (43). Expression (54) shows that the swap rate increases when the level of index variability increases. From this observation we can conclude that for a convex function $u$, the swap rate $\mathbb{E}\left[u\left(\frac{S}{\mathbb{E}[S]}\right)\right]$ could be interpreted as an indicator for the market perception concerning the variability of the stock market index price $S$ over the coming $T$ years. In Section 5.4.3, we will consider the particular choice of $u$ for which the approximation $\left[46\right.$ for $\mathbb{E}\left[\begin{array}{l}\left.S\left(\frac{S}{\mathbb{E}[S]}\right)\right] \\ \text { leads to }\end{array}\right.$ the well-known VIX formula, which is the current market standard for measuring market stress.

Notice however that some cautiousness is appropriate here, because the convex order in (54) is an order under the risk-neutral measure, whereas a statement about the variability of the stock market index is essentially a statement under the measure $\mathbb{P}$.

Taking into account the convex order relation (17)), we find from (54) and Jensen's inequality that the following inequalities hold for any convex function $u$ :

$$
u(1) \leq \mathbb{E}\left[u\left(\frac{S}{\mathbb{E}[S]}\right)\right] \leq \mathbb{E}\left[u\left(\frac{S^{c}}{\mathbb{E}[S]}\right)\right] .
$$

In Cheung et al. (2013) it was proven that if $u$ is strictly convex with absolutely continuous derivative $u^{\prime}$ and such that $\mathbb{E}\left[u\left(\frac{S^{c}}{\mathbb{E}[S]}\right)\right]$ is finite, the swap rate $\mathbb{E}\left[u\left(\frac{S^{c}}{\mathbb{E}[S]}\right)\right]$ fully characterizes a market with perfect herd behavior in the sense that

$$
\mathbb{E}\left[u\left(\frac{S}{\mathbb{E}[S]}\right)\right]=\mathbb{E}\left[u\left(\frac{S^{c}}{\mathbb{E}[S]}\right)\right] \Longleftrightarrow \underline{X} \text { is comonotonic. }
$$

Note that the expectations in $(56)$ are taken w.r.t the pricing measure $\mathbb{Q}$. As a result, the righ hand side of this equivalence relation is a statement under the pricing measure $\mathbb{Q}$. However, as comonotonicity is defined in terms of the support of the price vector $\underline{X}$ and moreover, $\mathbb{P}$ and $\mathbb{Q}$ are equivalent measures, we have that comonotonicity in the $\mathbb{Q}$-world is equivalent with comonotonicity in the $\mathbb{P}$-world.

The lower and upper bounds in (55) do not depend on the actual dependence structure of the price vector $\underline{X}$. Loosely speaking, the quantity $\mathbb{E}\left[u\left(\frac{S}{\mathbb{E}[S]}\right)\right]-u(1)$ reveals information about the dependence structure between the components of the price vector $\underline{X}$. Indeed, for given risk-neutral marginal distributions, we have that an increase of the co-movement between the components results in an increase of $\frac{S}{\mathbb{E}[S]}$ in convex order. From 54 , we find that this will also trigger an increase of the quantity $\mathbb{E}\left[u\left(\frac{S}{\mathbb{E}[S]}\right)\right]-u(1)$. The maximal value $\mathbb{E}\left[u\left(\frac{S^{c}}{\mathbb{E}[S]}\right)\right]-u(1)$ will be reached when the price vector $\underline{X}$ is comonotonic.

These observations lead to the conclusion that it may be reasonable to measure the $T$ year implied herd behavior by the ratio of $\left(\mathbb{E}\left[u\left(\frac{S}{\mathbb{E}[S]}\right)\right]-u(1)\right)$ and $\left(\mathbb{E}\left[u\left(\frac{S^{c}}{\mathbb{E}[S]}\right)\right]-u(1)\right)$, 
for an appropriate convex function $u$ :

$$
\text { ratio }=\frac{\mathbb{E}\left[u\left(\frac{S}{\mathbb{E}[S]}\right)\right]-u(1)}{\mathbb{E}\left[u\left(\frac{S^{c}}{\mathbb{E}[S]}\right)\right]-u(1)}
$$

provided $u(1)<\mathbb{E}\left[u\left(\frac{S^{c}}{\mathbb{E}[S]}\right)\right]<+\infty$. This ratio takes values between 0 and 1 , where a value of 1 represents the comonotonic market situation, whereas a value of 0 follows in case $S=\mathbb{E}[S]$.

Comonotonicity represents the situation where the stocks are non-compensating and there is no diversification possible. The situation where the price vector $\underline{X}$ satisfies $S=$ $\mathbb{E}[S]$ can be interpreted as a situation where there is perfect diversification. Indeed, the stocks are always perfectly compensating each other, resulting in no variability on the aggregate level. However, for given marginals, this lower bound is only reachable when the marginals are jointly mixable. The concept of joint mixability as an extreme negative dependence structure was discussed in Wang and Wang (2011), Wang et al. (2013) and Bernard et al. (2014).

In practice, only a limited number of strikes are traded, for the individual stocks as well as for the stock market index. In order to cope with this situation, we propose to replace the nominator and the denominator in (57) by the approximations (46) and (52), respectively. These considerations lead to the following definition for a first class of Herd Behavior Indices, based on swap rates.

Definition 2 (Herd Behavior Indices, based on swap rates) Consider the random vector $\underline{X}$ representing the stock prices $X_{i}, i=1,2, \ldots, n$ in $T$ years from now. The $T$-year implied Herd Behavior Index, based on the convex function $u:[0, \infty) \rightarrow \mathbb{R}$ with absolutely continuous derivative $u^{\prime}$, notation $\operatorname{HIX}_{u}[T]$, is defined by

$$
\operatorname{HIX}_{u}[T]=\frac{\mathrm{e}^{r T} \sum_{i=-l}^{h} u^{\prime \prime}\left(\frac{K_{i}}{\mathbb{E}[S]}\right) \Delta K_{i} Q\left[K_{i}\right]-\frac{u^{\prime \prime}\left(\frac{K_{0}}{\mathbb{E}[S]}\right)}{2}\left(\mathbb{E}[S]-K_{0}\right)^{2}}{\mathrm{e}^{r T} \sum_{i=-l}^{h} u^{\prime \prime}\left(\frac{K_{i}}{\mathbb{E}[S]}\right) \Delta K_{i} \bar{Q}^{c}\left[K_{i}\right]-\frac{u^{\prime \prime}\left(\frac{K_{0}}{\mathbb{E}[S]}\right)}{2}\left(\mathbb{E}[S]-K_{0}\right)^{2}},
$$

provided all $u^{\prime \prime}\left(\frac{K_{i}}{\mathbb{E}[S]}\right)$ are well-defined and finite, and the denominator is positive.

$\mathrm{HIX}_{u}[T]$ can be interpreted as a $T$-year forward looking measure for the degree of herd behavior. The nominator captures the real market situation. It is determined from observed index option prices, see (45) and (46). The denominator captures the comonotonic market situation and can be determined from observed stock option prices, see (52) and (53). As no distributional assumptions have to be made, $\mathrm{HIX}_{u}[T]$ is a model-free measure for the degree of herd behavior in the market. Monitoring this measure on a regular basis may give insight in the change of the market perception over time concerning co-movement behavior of the $T$-year prices of a given set of stocks. 


\subsection{Examples}

\subsubsection{HIX: the Herd Behavior Index}

In this subsection, we consider a particular member of the family of herd behavior measures based on swap rates, as introduced above. This measure was first proposed in Dhaene, Linders, Schoutens and Vyncke (2012), and was baptized the Herd Behavior Index (HIX).

The HIX corresponds to $\operatorname{HIX}_{u}[T]$ with the function $u$ given by

$$
u(x)=(x-1)^{2}, \quad x \geq 0 .
$$

From (57) it follows that HIX $[T]$ is based on an approximation of the following ratio:

$$
\text { ratio }=\frac{\operatorname{Var}[S]}{\operatorname{Var}\left[S^{c}\right]}
$$

Taking into account (58), HIX $[T]$ is defined by

$$
\operatorname{HIX}[T]=\frac{2 \mathrm{e}^{r T} \sum_{i=-l}^{h} \Delta K_{i} Q\left[K_{i}\right]-\left(\mathbb{E}[S]-K_{0}\right)^{2}}{2 \mathrm{e}^{r T} \sum_{i=-l}^{h} \Delta K_{i} \bar{Q}^{c}\left[K_{i}\right]-\left(\mathbb{E}[S]-K_{0}\right)^{2}} .
$$

The T-year HIX is the ratio of an index option-based estimate of the risk-neutral variance of the market index and a stock option-based estimate of the corresponding variance in the comonontonic market situation. The HIX can be interpreted as a scaled variance index, with a time-dependent scaling factor. The observed index option prices are used to describe the real market situation, while the theory of comonotonicity allows us to describe the extreme situation via the observed stock option quotes.

A numerical illustration of the 30 days Dow Jones based HIX for the period 2000 2009 can be found in Dhaene, Linders, Schoutens and Vyncke (2012). For the particular dates considered in Figures 1 and 2, the values of the 30 days Dow Jones based HIX (closing mid-prices) are presented in Table1.

Suppose now that $K_{0}$ is sufficiently close to the forward rate $\mathbb{E}[S]$, such that the term $\left(\mathbb{E}[S]-K_{0}\right)^{2}$ becomes negligible in the expression $(59)$. Furthermore, suppose that the distance between consecutive traded strikes is constant, i.e. $\Delta K_{i}=\Delta K_{j}, i, j=-l, \ldots, h$. Then we find the following (approximate) expression for the HIX:

$$
\operatorname{HIX}[T] \approx \frac{\sum_{i=-l}^{h} Q\left[K_{i}\right]}{\sum_{i=-l}^{h} \bar{Q}^{c}\left[K_{i}\right]} .
$$

In this case, the HIX is equal to the sum of all out-of-the-money index option prices, divided by the sum of all comonotonic out-of-the-money index option prices 


\subsubsection{DHIX: The Downside Herd Behavior Index}

Consider the convex function $u$ defined by

$$
u(x)=\left[(1-x)_{+}\right]^{2} .
$$

The herd behavior index associated with this function $u$ is called the Downside HIX (DHIX). We find that

$$
\operatorname{DHIX}[T]=\frac{2 \mathrm{e}^{r T} \sum_{i=-l}^{0} \Delta K_{i} P\left[K_{i}\right]-\left(\mathbb{E}[S]-K_{0}\right)^{2}}{2 \mathrm{e}^{r T} \sum_{i=-l}^{0} \Delta K_{i} \bar{P}^{c}\left[K_{i}\right]-\left(\mathbb{E}[S]-K_{0}\right)^{2}},
$$

provided that $K_{0}<\mathbb{E}[S]$. In case $K_{0}=\mathbb{E}[S], u^{\prime \prime}\left(\frac{K_{0}}{\mathbb{E}[S]}\right)$ is not defined. However, we propose to keep the formula for DHIX $[T]$ also in this case. The DHIX is based on out-ofthe money put options and can be considered as a measure for the extent to which stock prices will decline together. The formula for HIX $[T]$ contains out-of-the money call and put options; see (59), and is a measure for the extent to which stock price will move (up or down) together.

In case $K_{0}$ is sufficiently close to the forward rate $\mathbb{E}[S]$ and, moreover, $\Delta K_{i}=\Delta K_{j}$, $i, j=-l, \ldots, h$, the expression $(60)$ simplifies to

$$
\operatorname{DHIX}[T] \approx \frac{\sum_{i=-l}^{0} P\left[K_{i}\right]}{\sum_{i=-l}^{0} \bar{P}^{c}\left[K_{i}\right]} .
$$

In this case, DHIX is equal to the sum of all out-of-the-money index put option prices, divided by the sum of all comonotonic out-of-the-money index put option prices

\subsubsection{CIX: The Comonotonicity Index}

Until here, we assumed that $u$ is a real-valued function defined on $[0,+\infty) \longrightarrow \mathbb{R}$ with absolutely continuous derivative on $[0,+\infty)$. All previously derived expressions and approximations hold as well for any function $u:(0,+\infty)$ with absolutely continuous derivative on $(0,+\infty)$, provided we assume that any $T$ - year stock price $X_{i}$ takes values in $(0,+\infty)$, with probability 1 .

Let us illustrate this remark by considering the herd behavior index $\operatorname{HIX}_{u}[T]$ based on the function

$$
u(x)=-2 \ln x .
$$

This index, which is based on the following ratio:

$$
\text { ratio }=\frac{\mathbb{E}\left[\ln \left(\frac{S}{\mathbb{E}[S]}\right)\right]}{\mathbb{E}\left[\ln \left(\frac{S^{c}}{\mathbb{E}[S]}\right)\right]}
$$

was introduced in Dhaene, Dony, Forys, Linders and Schoutens $(2012)$, where the authors call it the Comonotonicity Index (CIX). 
From (58), it follows that CIX[T] is defined by

$$
\mathrm{CIX}[T]=\frac{2 \mathrm{e}^{r T} \sum_{i=-l}^{h} \frac{\Delta K_{i}}{K_{i}^{2}} Q\left[K_{i}\right]-\left(\frac{\mathbb{E}[S]-K_{0}}{K_{0}}\right)^{2}}{2 \mathrm{e}^{r T} \sum_{i=-l}^{h} \frac{\Delta K_{i}}{K_{i}^{2}} \bar{Q}^{c}\left[K_{i}\right]-\left(\frac{\mathbb{E}[S]-K_{0}}{K_{0}}\right)^{2}} .
$$

Considering the S\&P500 and supposing that $T=30$ days, one recognizes the VIX squared formula in the nominator of CIX[T]; see e.g. Chicago Board Options Exchange (2003). The methodology adopted by the CBOE can be generalized to other stock market indices and we will use the term VIX to denote the 30 days implied volatility of a given stock market index. The denominator can then be interpreted as the comonotonic VIX squared. This means that the CIX measures herd behavior by comparing the VIX squared with the maximal attainable value for the VIX squared, which is reached when all the underlying stocks are moving perfectly together; see also Whaley (2000) and Carr and $\mathrm{Wu}(2006)$.

A numerical illustration of the 30 days - CIX based on the Dow Jones can be found in Dhaene, Dony, Forys, Linders and Schoutens (2012). For the trading days August 21, October 23, November 20 and December 18, 2008, the corresponding values of the HIX, CIX, the ATM comonotonicity gap, as well as the VIX are given in Table1. One observes that the values for the HIX and the CIX are almost identical; for a motivation of this observation we refer to Dhaene, Linders, Schoutens and Vyncke (2012). Note also that the VIX in October 2008 is lower than the VIX in November 2008:

$$
V I X[\mathrm{Oct} 08]<V I X[\mathrm{Nov} 08] \text {, }
$$

whereas the opposite ordering holds for the HIX:

$$
H I X[\mathrm{Oct} 08]>H I X[\mathrm{Nov} 08] \text {. }
$$

Table 1: The Dow Jones based VIX, comonotonicity gap, HIX and CIX for the four different trading days.

\begin{tabular}{rrrrr}
\hline & apr-08 & Oct-08 & nov-08 & dec-08 \\
\hline Index price & 126.20 & 86.91 & 75.52 & 86.05 \\
VIX & $18.84 \%$ & $65.03 \%$ & $72.34 \%$ & $42.76 \%$ \\
ATM Com Gap & $61.09 \%$ & $82.90 \%$ & $80.39 \%$ & $75.58 \%$ \\
HIX & $36.95 \%$ & $74.35 \%$ & $64.89 \%$ & $58.73 \%$ \\
CIX & $36.53 \%$ & $72.84 \%$ & $63.34 \%$ & $56.39 \%$ \\
\hline
\end{tabular}

Finally, remark that the order of the VIX values is in correspondence with the convex ordering observed in Figure 1. 


\section{Distorted expectations and implied herd behavior}

\subsection{Distorted expectations in terms of observed option prices}

A distortion function is a non-decreasing function $g:[0,1] \rightarrow[0,1]$ such that $g(0)=0$ and $g(1)=1$. The distorted expectation of the stock index $S$, associated with distortion function $g$, notation $\rho_{g}[S]$, is defined by

$$
\rho_{g}[S]=\int_{0}^{+\infty} g\left(1-F_{S}(s)\right) \mathrm{d} s .
$$

Throughout this section we assume that the derivate of the distortion function $g$ is well-defined and finite on $[0,1]$, where a derivative in an endpoint has to be understood as a right or left derivative, and that $g^{\prime}$ is absolutely continuous on $[0,1]$. In this case, $\rho_{g}[S]$ is finite and can be expressed as

$$
\rho_{g}[S]=g^{\prime}(1) \mathbb{E}[S]-\int_{0}^{1}(1-q) g^{\prime \prime}(1-q) \operatorname{TVaR}_{q}[S] \mathrm{d} q,
$$

see e.g. Cheung et al. (2013).

From (10), it follows that one can rewrite the distorted expectation $\rho_{g}[S]$ as a mixture of TVaR's and LTVaR's:

$$
\begin{aligned}
\rho_{g}[S]= & g^{\prime}\left(1-F_{S}(\mathbb{E}[S])\right) \mathbb{E}[S]+\int_{0}^{F_{S}(\mathbb{E}[S])} q g^{\prime \prime}(1-q) \operatorname{LTVaR}_{q}[S] \mathrm{d} q \\
& -\int_{F_{S}(\mathbb{E}[S])}^{1}(1-q) g^{\prime \prime}(1-q) \operatorname{TVaR}_{q}[S] \mathrm{d} q .
\end{aligned}
$$

Taking into account (6) and (9), we find that

$$
\begin{aligned}
\operatorname{TVaR}_{q}[S] & =F_{S}^{-1}(q)+\frac{\mathrm{e}^{r T}}{1-q} C\left[F_{S}^{-1}(q)\right], \\
\operatorname{LTVaR}_{q}[S] & =F_{S}^{-1}(q)-\frac{\mathrm{e}^{r T}}{q} P\left[F_{S}^{-1}(q)\right],
\end{aligned}
$$

which holds for any $q \in(0,1)$. Inserting (65) and (66) in (64), and taking into account the well-known equivalence relation

$$
q \leq F_{S}(\mathbb{E}[S]) \Leftrightarrow F_{S}^{-1}(q) \leq \mathbb{E}[S],
$$

leads to an expression for $\rho_{g}[S]$ in terms of prices of put option with strikes smaller than or equal to $\mathbb{E}[S]$ and call options with strike larger than $\mathbb{E}[S]$. Loosely speaking, this is a model-free expression for $\rho_{g}[S]$ in terms of out-of-the-money option prices.

As before, we assume that the index option prices $C[K]$ and $P[K]$ can only be observed for a limited number of strikes, see (24). Here, we additionally assume that $K_{-l}=0$, and that $K_{h+1}=F_{S}^{-1}(1)$ is known and finite. 
In order to find a reasonable approximation for $\rho_{g}[S]$, based on the available option data, let us first approximate the partially known convex index call option curve $C$ by the piecewise linear convex function $\bar{C}$ connecting the observed points $\left(K_{j}, C\left[K_{j}\right]\right)$, for $j$ $=-l, \ldots, h+1$. Introducing the notation $\bar{S}$ for a r.v. with cdf determined by

$$
F_{\bar{S}}(s)=1+\mathrm{e}^{r T} \bar{C}^{\prime}[s+]
$$

we find that

$$
F_{\bar{S}}(s)=\left\{\begin{array}{cc}
0 & \text { if } s<0, \\
1+\mathrm{e}^{r T} \frac{C\left[K_{j+1}\right]-C\left[K_{j}\right]}{K_{j+1}-K_{j}} & \text { if } K_{j} \leq s<K_{j+1}, \\
1 & (j=-l, \ldots, h+1) \\
& \text { if } s \geq K_{h+1},
\end{array}\right.
$$

is the cdf that leads to the option curve $\bar{C}$. In the sequel, we always assume that $F_{\bar{S}}$ is strictly increasing in the traded strikes, in the following sense: if $F_{\bar{S}}\left(K_{j}\right)>0$, then $F_{\bar{S}}\left(K_{j}\right)>F_{\bar{S}}\left(K_{j-1}\right)$ for $j=-l, \ldots, h+1$, with $K_{-l-1}=-1$ by convention. If this condition is initially not satisfied, we decrease the set of observed points until it is satisfied.

From (67) one can determine the inverse of $F_{\bar{S}}$. Indeed, for any $j=-l, \ldots, h+1$, we find that $\overline{F_{\bar{S}}^{-1}}(q)$ is given by

$$
F_{\bar{S}}^{-1}(q)=K_{j}, \quad \text { if } F_{\bar{S}}\left(K_{j-1}\right)<q \leq F_{\bar{S}}\left(K_{j}\right) .
$$

Next, we propose to approximate the distorted expectation $\rho_{g}[S]$ by $\rho_{g}[\bar{S}]$. Similar to $(64), \rho_{g}[S]$ can be expressed as

$$
\begin{aligned}
\rho_{g}[\bar{S}]= & g^{\prime}\left(1-F_{\bar{S}}(\mathbb{E}[S])\right) \mathbb{E}[S]+\int_{0}^{F_{\bar{S}}(\mathbb{E}[S])} q g^{\prime \prime}(1-q) \operatorname{LTVaR}_{q}[\bar{S}] \mathrm{d} q \\
& -\int_{F_{\bar{S}}(\mathbb{E}[S])}^{1}(1-q) g^{\prime \prime}(1-q) \operatorname{TVaR}_{q}[\bar{S}] \mathrm{d} q .
\end{aligned}
$$

From $(6),(9)$ and $(68)$, we find that $\operatorname{TVaR}_{q}[\bar{S}]$ and $\operatorname{LTVaR}_{q}[\bar{S}]$ can be determined from the observed option curve. Indeed, for any $j=-l, \ldots, h+1$, they are given by

$$
\operatorname{TVaR}_{q}[\bar{S}]=K_{j}+\frac{\mathrm{e}^{r T}}{1-q} C\left[K_{j}\right], \quad \text { if } F_{\bar{S}}\left(K_{j-1}\right)<q \leq F_{\bar{S}}\left(K_{j}\right)
$$

and

$$
\operatorname{LTVaR}_{q}[\bar{S}]=K_{j}-\frac{\mathrm{e}^{r T}}{q} P\left[K_{j}\right], \quad \text { if } F_{\bar{S}}\left(K_{j-1}\right)<q \leq F_{\bar{S}}\left(K_{j}\right),
$$

respectively.

From $K_{0} \leq \mathbb{E}[S]<K_{1}$, it follows that $F_{\bar{S}}\left(\mathbb{E}_{[S])}\left[S F_{\bar{S}}\left(K_{0}\right)\right.\right.$. Inserting $(70)$ and $(71)$ in 69), we find an expression for $\rho_{g}[\bar{S}]$ in terms of traded out-of-the-money index options.

We introduce the following notation:

$$
p_{j}=F_{\bar{S}}\left(K_{j}\right), \quad j=-l,-l+1, \ldots, h+1 .
$$


Furthermore, we define $p_{-l-1}=0$. Note also that $p_{h+1}=1$ and $F_{\bar{S}}(\mathbb{E}[S])=p_{0}$. Furthermore, we define the constants $c_{j}$ and $d_{j}$ as follows:

$$
c_{j}=g^{\prime}\left(1-p_{j}\right)-g^{\prime}\left(1-p_{j-1}\right), \quad j=-l, \ldots, h+1,
$$

and

$$
d_{j}=\left\{\begin{array}{r}
p_{j-1} g^{\prime}\left(1-p_{j-1}\right)-p_{j} g^{\prime}\left(1-p_{j}\right)+g\left(1-p_{j-1}\right)-g\left(1-p_{j}\right), \\
j=-l, \ldots, 0 \\
\left(1-p_{j}\right) g^{\prime}\left(1-p_{j}\right)-\left(1-p_{j-1}\right) g^{\prime}\left(1-p_{j-1}\right)+g\left(1-p_{j-1}\right)-g\left(1-p_{j}\right), \\
j=1, \ldots, h+1
\end{array}\right.
$$

Note that the constants $c_{j}$ and $d_{j}$ are always well-defined and finite because we assumed that the derivative $g^{\prime}$ is always well-defined and finite in $[0,1]$. We can now rewrite the first integral of expression (69) as follows:

$$
\begin{aligned}
\int_{0}^{F_{\bar{S}}(\mathbb{E}[S])} q g^{\prime \prime}(1-q) \operatorname{LTVaR}_{q}[\bar{S}] \mathrm{d} q & =\sum_{j=-l}^{0} \int_{p_{j-1}}^{p_{j}} q g^{\prime \prime}(1-q) \operatorname{LTVaR}_{q}[\bar{S}] \mathrm{d} q \\
& =\sum_{j=-l}^{0} K_{j} \int_{p_{j-1}}^{p_{j}} q g^{\prime \prime}(1-q) \mathrm{d} q \\
& -\mathrm{e}^{r T} \sum_{j=-l}^{0} P\left[K_{j}\right] \int_{p_{j-1}}^{p_{j}} g^{\prime \prime}(1-q) \mathrm{d} q
\end{aligned}
$$

where we used expression (71) in the last step. Calculating the two integrals in expression (75) results in

$$
\int_{0}^{F_{\bar{S}}(\mathbb{E}[S])} q g^{\prime \prime}(1-q) \operatorname{LTVaR}_{q}[\bar{S}] \mathrm{d} q=\sum_{j=-l}^{0}\left(\mathrm{e}^{r T} P\left[K_{j}\right] c_{j}+K_{j} d_{j}\right) .
$$

Similarly, we can rewrite the second integral of expression (69) as follows:

$$
-\int_{F_{\bar{S}}(\mathbb{E}[S])}^{1}(1-q) g^{\prime \prime}(1-q) \operatorname{TVaR}_{q}[\bar{S}] \mathrm{d} q=\sum_{j=1}^{h+1}\left(\mathrm{e}^{r T} C\left[K_{j}\right] c_{j}+K_{j} d_{j}\right) .
$$

Combining these expressions, we find the following explicite expression for $\rho_{g}[\bar{S}]$

$$
\rho_{g}[\bar{S}]=g^{\prime}\left(1-p_{0}\right) \mathbb{E}[S]+\sum_{j=-l}^{h+1}\left(\mathrm{e}^{r T} Q\left[K_{j}\right] c_{j}+K_{j} d_{j}\right)
$$

where $Q$ is defined as follows:

$$
Q\left[K_{j}\right]=\left\{\begin{array}{ll}
P\left[K_{j}\right], & \text { if } j=-l, \ldots, 0 \\
C\left[K_{j}\right], & \text { if } j=1, \ldots, h+1 .
\end{array} .\right.
$$


We find that $\rho_{g}[\bar{S}]$ is a model-free approximation for $\rho_{g}[S]$, in terms of observed index option prices. Notice however that this approximation depends on the choice of the maximal strike $K_{h+1}$, because of the term $K_{h+1} d_{h+1}$ in the expression (76). The term $d_{h+1}$ is given by

$$
d_{h+1}=g\left(1-p_{h}\right)-\left(1-p_{h}\right) g^{\prime}\left(1-p_{h}\right) .
$$

Notice that $p_{h}$ depends on the choice of $K_{h+1}$ :

$$
p_{h}=1-\mathrm{e}^{r T} \frac{C\left[K_{h}\right]}{K_{h+1}-K_{h}} .
$$

The contribution of the term $K_{h+1} d_{h+1}$ in the expression (76) will be negligible for a sufficiently large $K_{h+1}$, provided

$$
\lim _{K_{h+1} \rightarrow+\infty} K_{h+1} d_{h+1}=0 .
$$

\subsection{Distorted expectations in case of perfect herd behavior}

Let us now consider the comonotonic market situation. In this case, the distorted expectation is given by $\rho_{g}\left[S^{c}\right]$, with $S^{c}$ defined in (16). Due to the finite number of traded stock option prices, see $(23)$, we cannot determine $\rho_{g}\left[S^{c}\right]$. Therefore, we propose to approximate it by $\rho_{g}\left[\bar{S}^{c}\right]$, with $\bar{S}^{c}$ defined in $(31)$. From the comonotonic additivity property of distorted expectations, it follows that

$$
\rho_{g}\left[\bar{S}^{c}\right]=\sum_{i=1}^{n} w_{i} \rho_{g}\left[\bar{X}_{i}\right]
$$

see e.g. Wang (1996) or Dhaene, Kukush, Linders and Tang (2012).

The distorted expectation $\rho_{g}\left[\bar{X}_{i}\right]$ corresponding to stock $i$ can be expressed as

$$
\begin{aligned}
\rho_{g}\left[\bar{X}_{i}\right] & =g^{\prime}\left(1-F_{\bar{X}_{i}}\left(\mathbb{E}\left[X_{i}\right]\right)\right) \mathbb{E}\left[X_{i}\right]+\int_{0}^{F_{\bar{X}_{i}}\left(\mathbb{E}\left[X_{i}\right]\right)} q g^{\prime \prime}(1-q) \operatorname{LTVAR}_{q}\left[\bar{X}_{i}\right] \mathrm{d} q \\
& -\int_{F_{\bar{X}_{i}}\left(\mathbb{E}\left[X_{i}\right]\right)}^{1}(1-q) g^{\prime \prime}(1-q) \operatorname{TVAR}_{q}\left[\bar{X}_{i}\right] \mathrm{d} q .
\end{aligned}
$$

Taking into account (6), (9) and (30), we find for any $j=-l_{i}, \ldots, h_{i}+1$, that

$$
\operatorname{TVaR}_{q}\left[\bar{X}_{i}\right]=K_{i, j}+\frac{\mathrm{e}^{r T}}{1-q} C_{i}\left[K_{i, j}\right], \quad \text { if } F_{\bar{X}_{i}}\left(K_{i, j-1}\right)<q \leq F_{\bar{X}_{i}}\left(K_{i, j}\right)
$$

and

$$
\operatorname{LTVaR}_{q}\left[\bar{X}_{i}\right]=K_{i, j}-\frac{\mathrm{e}^{r T}}{q} P_{i}\left[K_{i, j}\right], \quad \text { if } F_{\bar{X}_{i}}\left(K_{i, j-1}\right)<q \leq F_{\bar{X}_{i}}\left(K_{i, j}\right)
$$

From $K_{i, 0} \leq \mathbb{E}\left[X_{i}\right]<K_{i, 1}$, it follows that $F_{\bar{X}_{i}}\left(\mathbb{E}\left[X_{i}\right]\right)=F_{\bar{X}_{i}}\left(K_{i, 0}\right)$. Inserting (81) and 82 in (80) leads to an expression for $\rho_{g}\left[\bar{X}_{i}\right]$ in terms of traded out-of-the-money options on stock $i$. The value of $\rho_{g}\left[\bar{S}^{c}\right]$ can then be determined from 79 . 
One can prove that the distorted expectation $\rho_{g}\left[\bar{X}_{i}\right]$ can be determined in a modelfree way using observed stock option prices. Indeed, similar derivations than the one that have led to (76), result in

$$
\rho_{g}\left[\bar{X}_{i}\right]=g^{\prime}\left(1-p_{i, 0}\right) \mathbb{E}\left[X_{i}\right]+\sum_{j=-l_{i}}^{h_{i}+1}\left(\mathrm{e}^{r T} Q_{i}\left[K_{i, j}\right] c_{i, j}+K_{i, j} d_{i, j}\right),
$$

where the $Q_{i}$ and the constants $c_{i, j}$ and $d_{i, j}$ are defined in a similar way as in (77), (72) and (73), respectively.

\subsection{Measuring the degree of herd behavior in terms of distorted expectations}

Consider two points in time, $t$ and $t^{\prime}$, with $0 \leq t \leq t^{\prime}$. It is well-known that the following inequality holds for any concave distortion function:

$$
\frac{S}{\mathbb{E}[S]} \preceq_{c x} \frac{S^{\prime}}{\mathbb{E}^{\prime}\left[S^{\prime}\right]} \Longrightarrow \rho_{g}\left[\frac{S}{\mathbb{E}[S]}\right] \leq \rho_{g}\left[\frac{S^{\prime}}{\mathbb{E}^{\prime}\left[S^{\prime}\right]}\right],
$$

where we use the notations and conventions introduced in (27). For the special case of concave distortion functions with an absolute continuous derivate, this implication follows from (13) and (64). From (84), we can conclude that an increase in convex order of the forward deflated time- $T$ index price level leads to an increase of the corresponding

distorted expectation. Monitoring the quantity $\rho_{g}\left[\frac{S}{\mathbb{E}[S]}\right]=\frac{\rho_{g}[S]}{\mathbb{E}[S]}$ gives information about the changing variability of the $T$-year index price over time.

Taking into account the convex order relation (17), we find from (84) that for any concave distortion function the following inequalities hold:

$$
\mathbb{E}[S] \leq \rho_{g}[S] \leq \rho_{g}\left[S^{c}\right] .
$$

In Cheung et al. (2013) it was proven that if $g$ is a strictly concave distortion function, the distorted expectation $\rho_{g}\left[S^{c}\right]$ fully characterizes a market with perfect herd behavior:

$$
\rho_{g}[S]=\rho_{g}\left[S^{c}\right] \Longleftrightarrow \underline{X} \text { is comonotonic. }
$$

Loosely speaking, the difference $\rho_{g}[S]-\mathbb{E}[S]$ will increase when the co-movement between time- $T$ stock prices becomes stronger. It will reach its maximal value when the underlying price vector $\underline{X}$ is comonotonic. Therefore, in a distorted expectations framework, it may be reasonable to define the $T$-year implied herd behavior index as the ratio between the differences $\rho_{g}[S]-\mathbb{E}[S]$ and $\rho_{g}\left[S^{c}\right]-\mathbb{E}[S]$, for an appropriately chosen concave distortion function $g$ :

$$
\text { ratio }=\frac{\rho_{g}[S]-\mathbb{E}[S]}{\rho_{g}\left[S^{c}\right]-\mathbb{E}[S]}
$$


provided $\mathbb{E}[S]<\rho_{g}\left[S^{c}\right]$. This ratio takes values in $[0,1]$, where a value of 0 corresponds to the case where $\rho_{g}[S]=\mathbb{E}[S]$, while a value of 1 represents the comonotonic market situation. For given marginals, the value of 1 is always reachable, whereas a value of 0 is only reachable when the marginals are jointly mixable.

In order to cope with the finite number of traded strikes, we propose to replace $S$ and $S^{c}$ by the approximations $\bar{S}$ and $\bar{S}^{c}$, respectively. Taking into account the comonotonic additivity property (79), leads to the following definition for the Herd Behavior Index.

Definition 3 (Herd Behavior Index based on distorted expectations) Consider the random vector $\underline{X}$ representing the stock prices $X_{i}, i=1,2, \ldots, n$ at time $T$. The $T$-year implied Herd Behavior Index, based on the concave distortion function $g$ with absolute continuous derivative, notation $\mathrm{HIX}_{g}[T]$, is defined by

$$
\operatorname{HIX}_{g}[T]=\frac{\rho_{g}[\bar{S}]-\mathbb{E}[S]}{\sum_{i=1}^{n} w_{i} \rho_{g}\left[\bar{X}_{i}\right]-\mathbb{E}[S]},
$$

provided the denominator is positive.

The Herd Behavior Index defined in 87 can be determined from the available option prices. The nominator captures the real market situation. From (69), (70) and (71), it follows that it can be determined from traded index option prices. The denominator captures the comonotonic market situation and can be determined from available stock option prices, see (80), (81) and (82). As no assumption has to be made concerning the stock price dynamics, $\operatorname{HIX}_{g}[T]$ is a model-free measure for the degree of herd behavior in the market.

\subsubsection{Example}

A possible candidate for the distortion function $g$ is the MINVAR distortion function with parameter $\lambda$, as introduced in Cherny and Madan (2009):

$$
g(q)=1-(1-q)^{1+\lambda}, \quad q \in[0,1] \text { and } \lambda>0 .
$$

Note that $g$ is a concave distortion function and that its derivative $g^{\prime}$ is well-defined and finite in $[0,1]$. We have that $g^{\prime}(q)=(1+\lambda)(1-q)^{\lambda}$.

As an example, consider $\lambda=1$. The distorted expectation $\rho_{g}[\bar{S}]$ then follows from the expression (76) where the $c_{j}$ and $d_{j}$ are given by

$$
c_{j}=2\left(p_{j}-p_{j-1}\right), j=-l,-l+1, \ldots, h+1,
$$

and

$$
d_{j}=\left\{\begin{array}{c}
p_{j-1}^{2}-p_{j}^{2}, \quad j=-l, \ldots, 0 \\
\left(p_{j}-p_{j-1}\right)\left(2-p_{j}-p_{j-1}\right), \quad j=1, \ldots, h+1 .
\end{array}\right.
$$

Similar expressions can be derived for the distorted expectations $\rho_{g}\left[\bar{X}_{i}\right]$ of the individual stocks. 


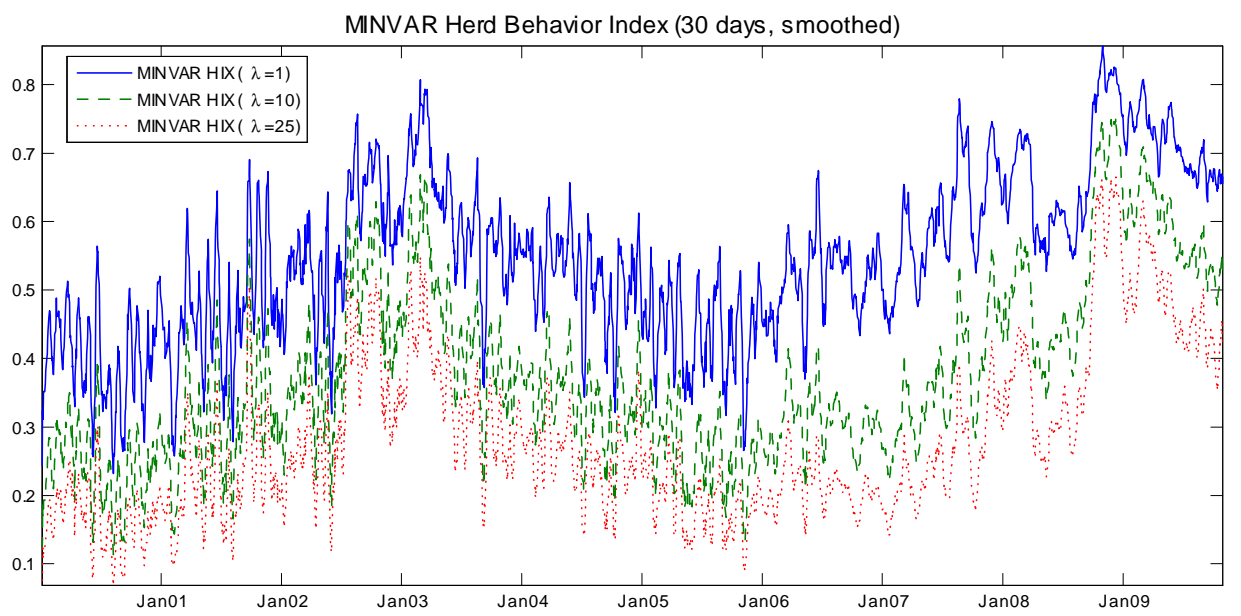

Figure 4: The smoothed MINVAR herd behavior index $H I X_{g}[30$ days $]$, where $g$ is the MINVAR distortion function with parameter $\lambda=1$ (solid line), $\lambda=10$ (dashed line) and $\lambda=25$ (dotted line).

For a MINVAR distortion function with parameter $\lambda=1$ we find that

$$
K_{h+1} d_{h+1}=\frac{\left(\mathrm{e}^{r T} C\left[K_{h}\right]\right)^{2}}{\left(\sqrt{K_{h+1}}-\frac{K_{h}}{\sqrt{K_{h+1}}}\right)^{2}},
$$

and hence $\lim _{K_{h+1 \rightarrow+\infty}} K_{h+1} d_{h+1}=0$. This means that the contribution of the term $K_{h+1} d_{h+1}$ in the expression (76) becomes negligible if $K_{h+1}$ is sufficiently large.

Consider the Dow Jones Industrial Average (DJ). Figure 4 shows the herd behavior index $D J-H I X_{g}[30$ days] for the MINVAR distortion function (88), with $\lambda=1$ (solid line), $\lambda=10$ (dashed line) and $\lambda=25$ (dotted line), based on closing midprices for the period January 2000 - October 2009. The graph is smoothed by taking at each trading day the average over the past 7 trading days. For a detailed description of the data and the numerical issues, we refer to Dhaene, Linders, Schoutens and Vyncke (2012). A comparison of the (smoothed) MINVAR herd behavior index with $\lambda=10$ and the Herd Behavior Index HIX $[T]$ defined in (59) is given in Figure 5

\section{Conclusion}

In this paper, we introduced several indices which can be used to measure the implied degree of co-movement between the time- $T$ prices of the stocks of a given stock market index. The definitions of these indices are based on a comparison between the real market situation, which can be observed via traded index option prices, and the synthetic market situation where all prices move perfectly together. The theory of comonotonicity allows us to construct this synthetic (non-observed) market situation from the observed stock 


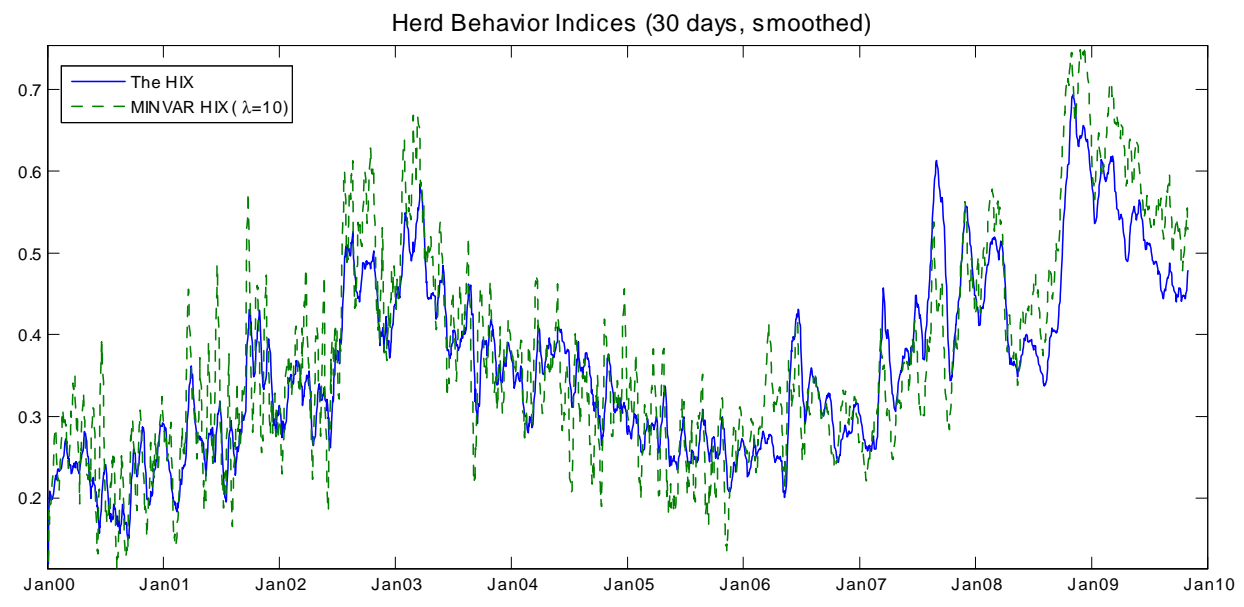

Figure 5: Smoothed version of the Herd Behavior Index HIX [30 days] and the MINVAR Herd Behavior Index $H I X_{g}[30$ days $]$ with parameter $\lambda=10$.

option prices. The comonotonicity gap refers to the distance between the observed and the synthetic index option curves. A small gap is a sign for a high degree of implied herd behavior, as we approach the situation where all prices move unison. A herd behavior index captures the comonotonicity gap in a number between 0 and 1 , where a value of 1 is equivalent with a comonotonic vector of time- $T$ stock prices, whereas a value of 0 is equivalent with a price vector where the stocks are always perfectly compensating.

We proposed two families of herd behavior measures. A first family is constructed by summarizing the index option curve in the swap rate $\mathbb{E}\left[u\left(\frac{S}{\mathbb{E}[S]}\right)\right]$, for an appropriate convex function $u$. This swap rate can be expressed as a combination of out-of-the-money index call and put options. Furthermore, this swap rate preserves convex order and its maximal value is attained when the underlying price vector is comonotonic. The swap rate $\mathbb{E}\left[u\left(\frac{S^{c}}{\mathbb{E}[S]}\right)\right]$, corresponding to the comonotonic situation, can be expressed in terms of stock option prices. The degree of herd behavior is then defined as the ratio of the (approximate) values of the swap rate and its comonotonic modification. The resulting measure is model-free, forward looking and can be determined from observed option data.

A second family of herd behavior measures is based on the observation that the distorted expectation $\rho_{g}[S]$, with concave distortion function $g$, can be expressed in terms of out-of-the-money index option data. Furthermore, such concave distorted expectations preserve convex order and attain their maximal value when the underlying price vector is comonotonic. The distorted expectation $\rho_{g}\left[S^{c}\right]$, corresponding to the comonotonic situation, can be expressed in terms of stock option prices. The degree of herd behavior is then defined as the ratio of the (approximate) values of the distorted expectation and its comonotonic modification. Again, the resulting index is model-free, forward looking and can be determined from available option data.

Acknowledgement: Daniël Linders, Jan Dhaene and Wim Schoutens acknowledge the financial support of the Onderzoeksfonds KU Leuven (GOA/13/002: Management 
of Financial and Actuarial Risks: Modeling, Regulation, Disclosure and Market Effects). Daniël Linders and Jan Dhaene also acknowledge the support of the AXA Research Fund (Measuring and managing herd behavior risk in stock markets).

\section{References}

Austing, P. (2014), Smile Pricing Explained, Financial Engineering Explained, Palgrave Macmillan.

Bekaert, G., Hodrick, R. J. and Zhang, X. (2009), 'International stock return comovements', The Journal of Finance 64(6), 2591-2626.

Bernard, C., Jiang, X. and Wang, R. (2014), 'Risk aggregation with dependence uncertainty', Insurance: Mathematics and Economics 54(1), 93 - 108.

Breeden, D. T. and Litzenberger, R. H. (1978), 'Prices of state-contingent claims implicit in option prices', Journal of Business 51(4), 621-51.

Carr, P., Geman, H., Madan, D. B. and Yor, M. (2011), 'Options on realized variance and convex orders', Quantitative Finance 11(11), 1685-1694.

Carr, P. and Madan, D. (2001), Towards a theory of volatility trading, in 'Option Pricing, Interest Rates and Risk Management', Cambridge University Press, pp. 458-476.

Carr, P. and Wu, L. (2006), 'A tale of two indices', The Journal of Derivatives 13(3), 1329.

Chen, X., Deelstra, G., Dhaene, J., Linders, D. and Vanmaele, M. (2015), 'On an optimization problem related to static super-replicating strategies', Journal of Computational and Applied Mathematics 278, 213-230.

Chen, X., Deelstra, G., Dhaene, J. and Vanmaele, M. (2008), 'Static super-replicating strategies for a class of exotic options', Insurance: Mathematics \& Economics 42(3), 1067-1085.

Cherny, A. and Madan, D. (2009), 'New measures for performance evaluation', Review of Financial Studies 22(7), 2571-2606.

Cheung, K. C., Dhaene, J., Kukush, A. and Linders, D. (2013), 'Ordered random vectors and equality in distribution', Scandinavian Actuarial Journal .

Chicago Board Options Exchange (2003), The CBOE volatility index - VIX, White Paper.

Chicago Board Options Exchange (2009), CBOE S\&P 500 implied correlation index, Working Paper.

Deelstra, G., Dhaene, J. and Vanmaele, M. (2011), An overview of comonotonicity and its applications in finance and insurance, in B. Oksendal and G. Nunno, eds, 'Advanced Mathematical Methods for Finance', Springer Berlin Heidelberg, pp. 155-179. 
Denuit, M., Dhaene, J., Goovaerts, M. and Kaas, R. (2005), Actuarial Theory for Dependent Risks: Measures, Orders and Models, John Wiley \& Sons, Ltd, Chichester, UK.

Dhaene, J., Denuit, M., Goovaerts, M., Kaas, R. and Vyncke, D. (2002a), 'The concept of comonotonicity in actuarial science and finance: theory', Insurance: Mathematics $\&$ Economics 31(1), 3-33.

Dhaene, J., Denuit, M., Goovaerts, M., Kaas, R. and Vyncke, D. (2002b), 'The concept of comonotonicity in actuarial science and finance: applications', Insurance: Mathematics E Economics 31(2), 133-161.

Dhaene, J., Dony, J., Forys, M. B., Linders, D. and Schoutens, W. (2012), Fix - the fear index: Measuring market fear, in 'Topics in Numerical Methods for Finance, Cummins M. et al. (eds.). Springer Proceedings in Mathematics and Statistics'.

Dhaene, J., Kukush, A., Linders, D. and Tang, Q. (2012), 'Remarks on quantiles and distortion risk measures', European Actuarial Journal 2, 319-328.

Dhaene, J., Linders, D., Schoutens, W. and Vyncke, D. (2012), 'The herd behavior index: A new measure for the implied degree of co-movement in stock markets', Insurance: Mathematics and Economics 50(3), 357-370.

Dhaene, J., Linders, D., Schoutens, W. and Vyncke, D. (2014), 'A multivariate dependence measure for aggregating risks', Journal of Computational and Applied Mathematics $\mathbf{2 6 3}(0), 78-87$.

Dhaene, J., Vanduffel, S., Goovaerts, M., Kaas, R., Tang, Q. and Vyncke, D. (2006), 'Risk measures and comonotonicity: a review', Stochastic Models 22, 573-606.

Dhaene, J., Wang, S., Young, V. and Goovaerts, M. J. (2000), 'Comonotonicity and maximal stop-loss premiums', Bulletin of the Swiss Association of Actuaries 2, 99 113.

Embrechts, P., McNeil, A. and Straumann, D. (1999), Correlation and dependence in risk management: properties and pitfalls, in 'Risk Management: Value at Risk and Beyond', Cambridge University Press, pp. 176-223.

Fonseca, J., Grasselli, M. and Tebaldi, C. (2007), 'Option pricing when correlations are stochastic: an analytical framework', Review of Derivatives Research 10(2), 151-180.

Garcia, J., Goossens, S., Masol, V. and Schoutens, W. (2009), 'Levy base correlation', Wilmott Journal 1, 95-100.

Harmon, D., De Aguiar, M. A., Chinellato, D. D., Braha, D., Epstein, I. and Bar-Yam, Y. (2011), 'Predicting Economic Market Crises Using Measures of Collective Panic', SSRN eLibrary .

Hobson, D., Laurence, P. and Wang, T. (2005), 'Static-arbitrage upper bounds for the prices of basket options', Quantitative Finance 5(4), 329-342. 
Kleykamp, D. and Liu, W.-C. (2012), 'Assessing co-movements in world equity markets', Tamkang Journal of International Affairs 16(1), 1-46.

Koch, I. and De Schepper, A. (2011), 'Measuring comonotonicity in m-dimensional vectors', ASTIN Bulletin 41, 191-213.

Laurence, P. (2008), A new tool for correlation risk management: the market implied comonotonicity gap, Global Derivatives, Paris, Invited Talk, May 2008.

Linders, D., Dhaene, J., Hounnon, H. and Vanmaele, M. (2012), Index options: a modelfree approach, Research report AFI-1265 FEB, Leuven: KU Leuven - Faculty of Business and Economics.

Linders, D. and Schoutens, W. (2014a), Basket option pricing and implied correlation in a Lévy copula model, Research report AFI-1494, FEB, KU Leuven.

Linders, D. and Schoutens, W. (2014b), 'A framework for robust measurement of implied correlation', Journal of Computational and Applied Mathematics 271, 39-52.

Madan, D. B. and Schoutens, W. (2013), 'Systemic risk tradeoffs and option prices', Insurance: Mathematics and Economics 52(2), 222-230.

Shaked, M. and Shanthikumar, J. G. (2007), Stochastic orders, Springer.

Skintzi, V. D. and Refenes, A. N. (2005), 'Implied correlation index: A new measure of diversification', Journal of Futures Markets 25, 171-197. doi: 10.1002/fut.20137.

Tavin, B. (2013), Hedging dependence risk with spread options via the power Frank and Power Student t copulas, Technical report, Université Paris I Panthéon-Sorbonne. Available at SSRN: http://ssrn.com/abstract=2192430.

Wang, B. and Wang, R. (2011), 'The complete mixability and convex minimization problems with monotone marginal densities', Journal of Multivariate Analysis 102(10), 1344 -1360 .

Wang, R., Peng, L. and Yang, J. (2013), 'Bounds for the sum of dependent risks and worst value-at-risk with monotone marginal densities', Finance and Stochastics 17(2), 395417.

Wang, S. (1996), 'Premium calculation by transforming the layer premium density', ASTIN Bulletin 26(1), 71-92.

Whaley, R. (2000), 'The investor fear gauge', Journal of Portfolio Management 26, 12-17. 
FACULTY OF ECONOMICS AND BUSINESS Naamsestraat 69 bus 3500

3000 LEUVEN, BELGIË

tel. + 3216326612

fax +3216326791

info@econ.kuleuven.be www.econ.kuleuven.be 